\title{
Envejecimiento
}

\section{de la población y sistemas de pensiones \\ en América Latina}

\section{Jorge Bravo}

División de Población,

Centro Latinoamericano

de Demografía (CELADE)

CEPAL

jbravo@eclac.cl
Este artículo examina el papel que desempeña el envejecimiento demográfico en la evolución de mediano a largo plazo de los sistemas de pensiones en América Latina y el Caribe. Está organizado en seis secciones. Luego de la sección introductoria, la sección II entrega información sobre las tendencias del envejecimiento y de los sistemas de pensiones en los países latinoamericanos, con algunas referencias al contexto internacional. La sección III presenta los indicadores, datos y métodos de análisis, y examina el efecto de las estructuras de población envejecidas y de la creciente longevidad en las variables de los sistemas: el gasto en pensiones, el balance financiero, las obligaciones pensionales y la rentabilidad implícita; también hace un análisis crítico de los criterios que suelen utilizarse para evaluar la sustentabilidad de los sistemas de reparto. Luego, la sección IV introduce de manera más sistemática el concepto y los indicadores de la deuda personal implícita, entrega estimaciones de la magnitud de las obligaciones pensionales que deben hacerse explícitas al cambiar a un régimen de capitalización, y analiza los efectos de la estructura etaria y de la mortalidad adulta en los países latinoamericanos. La sección $\mathrm{V}$ examina brevemente los costos fiscales de transición que es preciso afrontar cuando se aplica este tipo de reforma, y por último, la sección VI proporciona un resumen y conclusiones. 


\section{I}

\section{Introducción}

La región de América Latina y el Caribe ha experimentado cambios demográficos sustanciales durante las últimas décadas, que han modificado la base de la población y el funcionamiento de las instituciones que transfieren recursos entre grupos generacionales. Una particular expresión de estos cambios demográficos, el envejecimiento de la población, está relacionada directamente con la evolución de los sistemas de seguridad social y, en la medida que la tendencia al envejecimiento se intensifique, sus efectos se expandirán y amplificarán, como lo demuestra muy claramente la experiencia histórica de los países más desarrollados.

Este es el punto de vista fundamental de este estudio, que examina el papel que desempeña el envejecimiento demográfico en la evolución a mediano y largo plazo de los sistemas de pensiones. Esta síntesis se basa en trabajos realizados en los últimos años en el CELADE y en colaboración con la Unidad de Financiamiento para el Desarrollo de la CEPAL, sobre los aspectos demográficos y financieros de las reformas de los sistemas de pensiones en la región.

El análisis se aboca en primer lugar a examinar los efectos de los cambios en la estructura etaria y en la mortalidad de la población adulta sobre algunos indicadores de los sistemas de reparto. En seguida se ocupa de las transiciones de los sistemas de reparto a los de capitalización, y en particular de la contribución de las variables demográficas a la determinación de las obligaciones de pensiones y los costos fiscales de transición. Con ello, el artículo aúna y analiza varios hechos y resultados conocidos de la investigación aplicada, y utiliza las proyecciones de mediano y largo plazo para examinar los límites a los que pueden extenderse estas relaciones.

\footnotetext{
$\square$ La versión original de este artículo se elaboró mientras el autor era consultor principal del programa CEPAL-CELADE/BID, ATN/TF-5827RG, durante una estada en el Institut national d'études démographiques (INED) en París, Francia, del 15 de diciembre de 1998 al 15 de febrero de 1999, facilitada por el programa de relaciones internacionales del instituto. La revisión, realizada en la CEPAL, contó con las valiosas observaciones de Didier Blanchet, Georges Tapinos, Antoine Bommier, Andras Uthoff, Juan Chackiel, Carmelo MesaLago, Mario Marcel, y dos lectores anónimos del BID. El autor es el único responsable de cualquier error o deficiencia remanente.
}

Se ha procurado examinar estos temas de manera sistemática, pero hay muchas cuestiones importantes que no se abordan en esta presentación. Entre ellas, no se tratan las relaciones de equilibrio general, salvo ciertas referencias hechas en relación con tópicos específicos. Muchos aspectos que suscitan debates importantes, como los que confrontan los sistemas de reparto con los de capitalización, los sistemas administrados por el Estado con los de administración privada, sólo se consideran en la medida que contribuyen al conocimiento de las relaciones de especial interés para el artículo. ${ }^{1}$ Los efectos de la diferenciación de género, que se vuelven más importantes con el envejecimiento de la población, es otro tema destacado que aquí no se examina (véanse Arenas, 2000; Cox, 1999; Bravo, 1999).

Sin embargo, el enfoque más específico que se adopta aquí permite un conocimiento y presentación más claros de los efectos demográficos sobre varias dimensiones de los sistemas de pensiones. En consecuencia, el artículo se organiza como sigue. La sección II ofrece antecedentes sobre las tendencias del envejecimiento y de los sistemas de pensiones en los países latinoamericanos, con cierta referencia al contexto internacional. La sección III justifica el enfoque analítico, el modelo que se utiliza, y examina el efecto de las estructuras de población envejecidas y de la mayor supervivencia sobre cuatro variables importantes del sistema de reparto: el gasto en pensiones, el balance financiero del sistema, las obligaciones por concepto de pensiones y la rentabilidad implícita del sistema. Esta sección brinda también un análisis crítico de los criterios utilizados para calificar la sustentabilidad de los sistemas de reparto.

La sección IV introduce de manera más sistemática el concepto y las mediciones de la deuda pensional implícita, y presenta estimaciones de la magnitud de las obligaciones que deben hacerse explícitas al pasar a un régimen de capitalización, así como un análisis

\footnotetext{
${ }^{1}$ Un buen aporte a ese debate sería un análisis integral de las virtudes y limitaciones más importantes de los diferentes esquemas, que incluya los múltiples aspectos demográficos, económicos y políticos pertinentes, así como las estrategias de desarrollo de los países en que va a aplicarse la evaluación. Esto escapa con mucho al alcance del presente estudio.
} 
de los efectos de la estructura etaria y la mortalidad adulta en los países latinoamericanos. La sección V examina someramente los costos fiscales transicionales que hay que encarar cuando se aplica este tipo de reforma, y la sección VI contiene el resumen y las observaciones finales.

\section{II \\ El envejecimiento de la población y los sistemas de pensiones: América Latina en el contexto internacional}

Tras experimentar las primeras etapas de la transición demográfica, asociadas con reducciones de la mortalidad en la niñez y disminuciones importantes de la fecundidad, la población de América Latina está comenzando a envejecer. Las personas mayores de 60 años constituyen el grupo de población de más rápido crecimiento en la región: su tamaño actual de unos cuarenta millones probablemente se duplicará dentro de unos veinte años. La proporción de la población mayor de 60 años es todavía reducida en comparación con la de los países más desarrollados: constituye hoy casi 8\% de la población en América Latina, comparado con un $16 \%$ en los Estados Unidos, 20\% en Europa, y casi $23 \%$ en Japón (Naciones Unidas, 1998). No obstante, la mortalidad en la región ha venido disminuyendo sustancialmente durante este siglo y ahora es relativamente baja en muchos países; el promedio de la esperanza de vida al nacer ya ha alcanzado la marca de los setenta años, y la esperanza de vida a los 60 años es de casi 20 años (CEPAL/CELADE, 1998) en la región en su conjunto. En casi todos los países de la región, las reducciones de la fecundidad tienden a seguir las reducciones de la mortalidad con un desfase temporal relativamente breve, menos de dos décadas en la mayoría de los casos (Bravo, 1992). Consideradas en conjunto, estas tendencias implican que el envejecimiento, medido por la proporción "vieja" en la población o por la relación de dependencia de la vejez, se va a acelerar durante las próximas décadas, y lo va a hacer a una velocidad mucho mayor que la que se registró en las sociedades actualmente más envejecidas. Se espera que los cambios en estos indicadores, que tardaron seis a diez décadas en producirse en los países desarrollados, tarden sólo dos a tres décadas en la mayoría de los países latinoamericanos. ${ }^{2}$
Tal como sucede con casi todas las variables socioeconómicas y demográficas en la región, existe una gran heterogeneidad entre países con respecto al envejecimiento: algunos tienen, y seguirán teniendo por muchos años más, distribuciones etarias bastante jóvenes (por ejemplo, Nicaragua, Honduras, Bolivia). Un número importante está en las etapas intermedias de la transición demográfica y se proyecta que envejecerán significativamente durante las próximas décadas (incluyendo los dos más poblados, Brasil y México), mientras que otros están ya relativamente envejecidos, como Uruguay, Argentina y algunos del Caribe. Países como Chile, Costa Rica y Panamá están un poco menos envejecidos, ya que tienen niveles reducidos de mortalidad, pero una fecundidad no tan baja todavía. Cuba es un caso especial, pues siendo el país de la región donde la fecundidad ha declinado más rápida y acentuadamente desde los años sesenta, se transformará en la nación más envejecida en poco más de una década. El envejecimiento varía también dentro de los países, entre los grupos socioeconómicos y según el género. Tal como se documenta en detalle en otros estudios, el contexto social específico, así como la particular secuencia de acontecimientos durante la transición demográfica, también se diferencia de un país a otro (Guzmán, 1996; Cosio-Zavala, 1996). Los diversos contextos demográficos se tomarán en cuenta en el análisis que sigue, en la medida que incidan en los patrones del envejecimiento.

En cuanto a los sistemas públicos de pensiones que se analizan aquí en relación con el envejecimiento demográfico, interesa recordar que no son en todas

\footnotetext{
2 Para análisis más extensos de las tendencias del envejecimiento en la región, véanse Chackiel (2000), y Villa y Rivadeneira (2000).
} 
partes ni siempre los medios dominantes para brindar apoyo económico a la población en edad avanzada. Las transferencias intergeneracionales, realizadas mediante mecanismos familiares/comunitarios o de mercado (Sauvy, 1953; Lee, 1995), han sido tradicionalmente importantes, y siguen siéndolo tanto en los países en desarrollo como desarrollados. ${ }^{3}$ En América Latina, algunos países habían comenzado la institucionalización de sus sistemas de pensiones a comienzos del siglo XX (como Argentina, Chile y Uruguay), pero la mayoría de los sistemas nacionales se volvieron más generalizados en el período de la posguerra. Desde su creación, los sistemas han expandido su cobertura y han aportado beneficios importantes a una fracción significativa de la población en edad avanzada. Los sistemas sufrieron también una serie de problemas. Ha habido dificultades para alcanzar el ideal de la cobertura universal, asegurar una recaudación completa y oportuna de las contribuciones, proteger el valor real de los fondos de reserva, y realizar ajustes en las contribuciones y los beneficios requeridos por los cambios demográficos y la maduración de los sistemas (MesaLago, 1985; Uthoff, 1997). Los problemas se acumularon con los años y se acentuaron con la crisis económica de los años ochenta en la región, lo que hizo más evidentes y visibles las deficiencias administrativas y financieras. Esos hechos influyeron considerablemente en la evaluación de los sistemas de pensiones y en las propuestas de reformas que surgieron durante esa década y se materializaron durante los años noventa. En las secciones siguientes examinaremos algunos aspectos de la reforma, desde la perspectiva del envejecimiento demográfico y sus implicaciones sobre el financiamiento y la distribución intergeneracional.

En América Latina, como en el resto del mundo, la mayoría de los sistemas públicos de pensiones son de reparto y cuentan con reservas relativamente escasas. ${ }^{4} \mathrm{El}$ gasto público en pensiones ha crecido consis-

\footnotetext{
${ }^{3}$ Los datos examinados por Lee (1995) indican que las transferencias intergeneracionales en las sociedades tradicionales son por lo general "descendentes", es decir, de los miembros más viejos a los más jóvenes de la población. Esto también se cumple en el caso de las transferencias familiares en los países desarrollados contemporáneos. Es la fuerte dirección "ascendente" de las transferencias encauzadas a través de la acumulación de ahorro y capital y el sector público (incluidas las pensiones) lo que produce, como resultado neto, transferencias intergeneracionales ascendentes en las sociedades más desarrolladas.

${ }^{4}$ Una excepción notable la constituye Estados Unidos, donde el importante fondo del seguro de vejez, enfermedad y muerte (OASDI) seguirá creciendo durante por lo menos otra década, en previsión de la jubilación de grandes cohortes emanadas del auge de nacimientos de principios de los años sesenta. Sin embargo, hay una gran controversia respecto a la liquidez y real valor económico de los activos que integran este fondo.
}

tentemente a escala mundial, y ha alcanzado en promedio entre $8 \%$ y $10 \%$ del PIB en la Organización de Cooperación y Desarrollo Económicos (OCDE) y en los países de Europa oriental (Banco Mundial, 1994; Dumont, 1998). En muchos países industriales, el gasto en seguridad social figura entre los dos o tres principales gastos de gobierno; la seguridad social en los Estados Unidos es actualmente el programa de gobierno más importante, ya que superó al de la defensa a comienzos de los años noventa. Las proyecciones de la OCDE (Leibfritz y Roseveare, 1995) prevén que en el siglo XXI los pagos de pensiones podrían fluctuar entre $5 \%$ y $20 \%$ del PIB, y que los déficit podrían fluctuar entre $0 \%$ y $10 \%$ del PIB, si no se realizan ajustes sustanciales en los próximos años. En estas tendencias proyectadas inciden sobre todo la tendencia de largo plazo del envejecimiento demográfico y las fluctuaciones proyectadas en la estructura por edades de la población producidas por el auge de nacimientos del inicio de los años sesenta.

En cambio, América Latina muestra un nivel global de gasto en pensiones similar al de Asia, ${ }^{5}$ del orden del $2 \%$ a $3 \%$ del PIB en promedio. La comparación de este nivel agregado de gasto con los indicadores de envejecimiento citados previamente revela una brecha importante entre los países latinoamericanos y las regiones y países más desarrollados, que tienen indicadores de envejecimiento que duplican o triplican los de América Latina, pero que gastan cuatro o cinco veces más en pensiones, en relación con su PIB. Retomaremos el análisis de estas diferencias en la sección siguiente.

Antes de pasar a una evaluación más detenida de los efectos del envejecimiento, conviene dar una ojeada a la perspectiva global de este fenómeno en las décadas venideras. Como se ilustra en el gráfico 1, se espera que en todas las regiones del mundo se acelere el proceso de envejecimiento de los adultos (medido por la relación de dependencia de la vejez) durante las próximas décadas, incluso en las poblaciones ya envejecidas de Europa y América del Norte. Estos cambios son bastante considerables: mientras en 1990 la relación de dependencia de la vejez (población de 65 y más años respecto a la de 15 a 64 años) variaba entre $6 \%$ y $18 \%$ entre regiones (promedio mundial $=10 \%$ ), se proyecta que en el 2030 todas las regiones, excepto

\footnotetext{
${ }^{5}$ No obstante, hay varias diferencias entre las dos regiones: en Asia, los sistemas públicos son en general de creación más reciente, y tienden a cubrir una proporción menor de la fuerza laboral y de las personas de edad (Kinsella y Gist, 1995).
} 
África, tendrán relaciones de dependencia que oscilarán entre $16 \%$ y $35 \%$ (promedio mundial $=17 \%$ ). ${ }^{6}$

El gráfico indica que la población de América Latina y el Caribe envejecerá con mayor rapidez que el promedio, puesto que se proyecta que en ella la relación de dependencia de la vejez alcanzará el promedio mundial en el 2030, pero a partir de una cifra inferior al promedio (8\%) en 1990, y que en el 2050 la relación de dependencia latinoamericana se triplicará con creces para llegar a cerca de $27 \%$, unos tres puntos porcentuales sobre el promedio mundial. La intensidad de esta tendencia proyectada sólo es sobrepasada por las proyecciones de largo plazo para los países europeos y de la OCDE, que muestran los efectos más sustanciales del envejecimiento sobre los sistemas de pensiones estimados hasta ahora (Roseveare y otros, 1996; Calot y Chesnais, 1997).

Cuando estos cambios ocurren en contextos de maduración de los sistemas, con restricciones del mercado laboral que se reflejan en un desempleo elevado y bajo crecimiento salarial, es evidente que las tenden-

GRAFICO 1

Perspectivas de envejecimiento en el mundo, por región, 1990-2050

(Población mayor de 65 años/población entre 15 y 64 años)

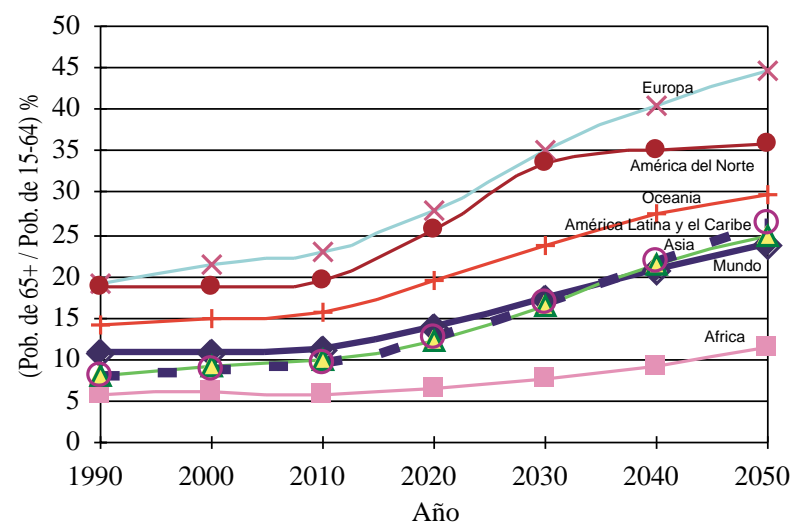

Fuente: Naciones Unidas (1998). cias demográficas acentúan los efectos económicos adversos y los problemas estructurales existentes que afectan los sistemas públicos. Sin embargo, es sin duda incorrecto, como se observará en las secciones siguientes, interpretar estas dificultades como prueba de que las tendencias demográficas hacen insustentables estos sistemas, y que implican la "bancarrota" o una crisis insuperable. En el mismo tenor, los países pueden complementar los sistemas de reparto existentes administrados por el gobierno con sistemas financiados y administrados por entidades privadas, $\mathrm{u}$ otros componentes (mixtos), como algunos lo hacen actualmente. Pero existen numerosos estudios y diversas experiencias de países en todo el mundo que dejan en claro ${ }^{7}$ que las tendencias demográficas ya citadas no demuestran que la capitalización plena es necesariamente la única solución o la más conveniente para los problemas de los sistemas de pensiones, como plantean algunos observadores. Actualmente, la mayoría de los analistas concuerda en que los estudios y evaluaciones de política cabales deben considerar diferentes opciones, que incluyen diversos grados de reparto y capitalización, sistemas definidos por beneficios y por contribuciones, y combinaciones de participación pública y privada (Mesa-Lago, 1994; CEPAL, 1998; Thompson, 1999; Orszag y Stiglitz, 1999; Holzmann, 1999), tomando en cuenta las diferentes condiciones nacionales, tanto demográficas como económicas y políticas.

Las siguientes secciones de este estudio examinan las tendencias de mediano plazo de los sistemas desde una perspectiva demográfica amplia, analizando en primer lugar varios indicadores de los sistemas de pensiones y su relación con el envejecimiento. A continuación se consideran las obligaciones vinculadas a las pensiones en los sistemas de reparto, y el papel que desempeñan en ellas la estructura etaria de la población y la mortalidad en edad avanzada. Más adelante se abordan los costos fiscales emanados de las transiciones del sistema de reparto al de capitalización, para luego concluir con observaciones finales.

\footnotetext{
${ }^{6}$ Una interpretación sencilla de esta tendencia en términos de las variables del sistema de pensiones es que, sólo por concepto del envejecimiento de la población, las tasas de cotización tendrían que aumentar en un $70 \%$ durante este período para que el balance financiero no empeorara con respecto a sus valores de 1990.
}

\footnotetext{
${ }^{7}$ Véanse, entre muchos otros, Blanchet, 1990; CEPAL/CELADE, 1996; Chand y Jaeger, 1996; Conseil d'analyse économique, 1998; ConteGrand, 1995; Reynaud, coord., 1998, y CEPAL, 1998.
} 


\section{III}

\section{Indicadores de los sistemas de pensiones a lo largo del proceso de envejecimiento}

\section{Indicadores, datos y métodos de análisis}

Casi todos los indicadores de los sistemas de pensiones están influidos por variables demográficas, sobre todo y en forma más directa por el envejecimiento de la población. En este artículo hemos escogido cuatro variables para ilustrar nuestro análisis de los efectos del envejecimiento sobre diferentes aspectos de los sistemas de pensiones de reparto: i) el gasto anual en pensiones, expresado como porcentaje del PIB; ii) el balance anual del sistema de pensiones — es decir, ingresos menos gastos-, que puede expresarse como una fracción de los ingresos del sistema o del PIB; iii) la obligación pensional implícita denominada también deuda pensional implícita, ${ }^{8}$ y iv) la rentabilidad implícita (o interna) del sistema, que corresponde a los flujos de los pagos de contribuciones y de las sumas percibidas por concepto de pensiones durante la vida de una cohorte. En los apéndices 1 y 2 figuran más detalles sobre los conceptos, medidas y datos utilizados para su cálculo.

Los indicadores más estándares, como el gasto anual en pensiones o el balance financiero del sistema, son mediciones periódicas (es decir, flujos anuales) que sirven para cuantificar la gravitación del sistema de pensiones sobre la economía nacional, pero que dan poca información sobre la sustentabilidad financiera de mediano o largo plazo del sistema o de su desempeño distributivo, en particular en diferentes grupos generacionales. Estos últimos aspectos los captan mejor, aunque de manera parcial e imperfecta, otros indicadores resumen de los flujos de contribuciones y beneficios a través del ciclo de vida. Dos de éstos son la deuda pensional implícita y la rentabilidad implícita del sistema, que se analizan en las secciones siguientes. Hemos escogido $d$, la relación de dependencia de

\footnotetext{
${ }^{8}$ Como se explica con mayor detalle más adelante, en la sección IV, hay varias medidas posibles de las obligaciones previsionales en los sistemas de reparto; en este estudio utilizamos el valor presente de las pensiones futuras que percibirán los pensionados y de las contribuciones ya realizadas por la población económicamente activa en un momento dado, expresados como porcentaje del PIB.
}

la vejez, ${ }^{9}$ que en adelante llamaremos simplemente relación de dependencia, como el indicador del envejecimiento demográfico para el análisis de los indicadores seleccionados del sistema de pensiones. El envejecimiento de individuos o cohortes de poblaciones, medido por el incremento de la esperanza de vida, tiende a preceder y luego a superponerse históricamente con los cambios en $d$, aunque la correlación entre estas dos formas de envejecimiento no es estricta, y sus consecuencias sobre los sistemas de pensiones son de índole y alcance diferentes, como se mostrará en las secciones siguientes.

La sección A del gráfico 2 muestra los valores observados del gasto en pensiones como porcentaje del PIB, estimados sobre la base de los datos compilados por la оाт (1997) del gasto nacional en seguridad social en el período 1991-1993. Esas estimaciones se refieren a las pensiones de vejez, supervivencia e invalidez del sector público y privado, y excluyen las transferencias hechas a otros programas. Deben considerarse sólo como el reflejo de órdenes de magnitud, puesto que la cifra precisa puede variar según los criterios específicos de medición que se utilicen. Asimismo, no se dispone de información sobre Brasil, México, Perú y Uruguay, cuatro países de la región muy importantes.

No obstante, los datos, graficados frente a la proporción de la población de 60 años y más, sugieren un par de cosas. Primero, como ya se había previsto, un gran número de países tiene un gasto del orden de $2 \%$ del PIB o menos; entre ellos, un número apreciable de países caribeños y centroamericanos, Ecuador y Bolivia. Los países restantes tienen un gasto que va desde un mínimo cercano a 3\% en Colombia a un máximo de más de $8 \%$ del PIB en Cuba; en un nivel intermedio están países como Argentina, Panamá y Chile. Considerados en su conjunto, estos datos revelan niveles de gasto bajos a medianos, según los estándares internacionales. Segundo, aunque varios de los países con

\footnotetext{
${ }^{9}$ Para los fines de este trabajo, de aquí en adelante, dicha relación se establece entre la población de 60 años y más y la de 20 a 59 años.
} 
GRAFICO 2

\section{América Latina y el Caribe (algunos países): Gasto y balance financiero de los sistemas de pensiones, según grado de envejecimiento, 1990-1993}

A. Gasto en pensiones y envejecimiento de la población

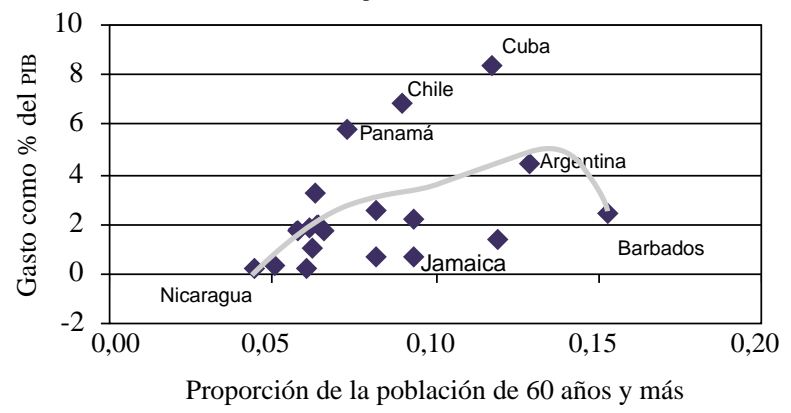

B. Balance financiero y relación de dependencia de la vejez.
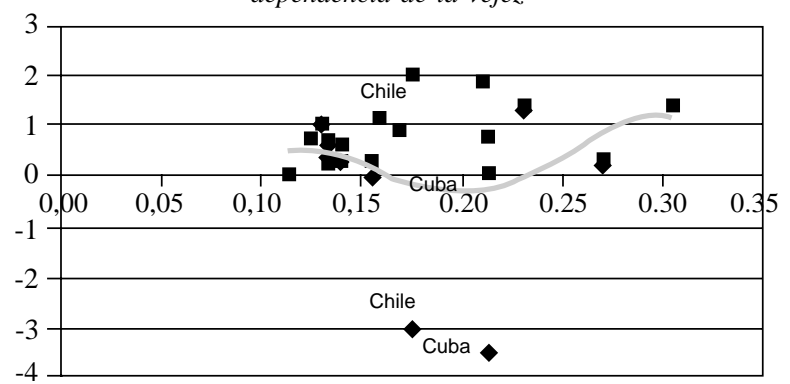

- Incluye contribución del Estado $\bullet$ Excluye contribución del Estado

Fuente: Estimaciones del autor, basadas en OIT (1997), CEPAL/ CELADE (1996) y Naciones Unidas (1999).

gasto elevado están en las categorías de envejecimiento mediano a elevado dentro de la región, no hay una relación estrecha manifiesta entre el grado de envejecimiento y el gasto en pensiones. Esto refleja en parte los problemas de comparabilidad de los datos de gasto, pero probablemente lo que es más importante, diferencias en la cobertura de la población del sistema de pensiones, las tasas de cotización y reemplazo, las condiciones de elegibilidad y el grado de maduración.

Estos dos tipos de limitaciones están presentes, al menos en el mismo grado, en el análisis del balance financiero del sistema. A los problemas de comparabilidad en el gasto, deben agregarse los asociados con los ingresos, que se ven afectados por diferencias internacionales en la definición de los componentes y programas del sistema, y en las convenciones contables, especialmente las relativas al tratamiento de las transferencias provenientes de otros programas, así como a la contribución estatal descontada la contribución del sector público como empleador (véase el apéndice 1). Teniendo presente estas advertencias, las estimaciones del balance del sistema presentadas en la sección B del gráfico 2 sugieren que el balance superavitario es la norma, especialmente en los países con sistemas más incipientes. Las excepciones son el pequeño déficit de Panamá y los más sustanciales de Chile y Cuba. ${ }^{10}$ Estos dos últimos países son casos especiales: las cuentas de ingresos y gastos de Chile están muy afectadas por el déficit de transición que emana de su reforma hacia el régimen de capitalización, y las de Cuba por el hecho de que el Estado absorbe el desequilibrio primario, de modo que su sistema aparece exactamente equilibrado cuando se incluyen las contribuciones estatales. La relación de corte transversal observada entre la relación de dependencia demográfica y el balance es incluso más débil que en el caso del gasto, por razones similares a las ya expuestas.

En suma, los datos recién analizados dan una información general útil sobre los indicadores y las relaciones de interés, pero ofrecen posibilidades más bien limitadas para su estudio más detallado y específico. ${ }^{11}$ Con el fin de aislar y analizar los efectos del envejecimiento sobre los sistemas de pensiones, un enfoque más viable y productivo consiste en utilizar modelos de nivel nacional, pero desagregados según edad, que tomen en cuenta los parámetros claves del sistema. El modelo siguiente, que necesita hacer abstracción de varios de los detalles y peculiaridades de los sistemas (véase el apéndice 2), da órdenes de magnitud aproximados de los indicadores de interés, y no estimaciones precisas. ${ }^{12} \mathrm{El}$ costo que supone la simplificación provee como compensación, adicionalmente, la posibilidad de estudiar una gama más amplia de niveles de envejecimiento (más allá de los observados hasta aho-

\footnotetext{
10 Argentina y Uruguay también tenían déficit considerables de la seguridad social a fines de los años setenta y comienzos de los ochenta (Mesa-Lago, 1991, cuadro 9).

${ }^{11}$ En principio, otro enfoque sería realizar análisis longitudinales, para países con series cronológicas confiables de datos sobre gasto e ingreso. Esto requiere series estadísticas consistentes y completas que abarquen al menos cuatro o cinco décadas, a fin de que los indicadores del envejecimiento presenten variaciones sustanciales, lo que impone una restricción adicional a la posibilidad de practicar este tipo de análisis para la mayoría de los países latinoamericanos. 12 Las estimaciones son estrictamente válidas, si son constantes las reglas del sistema, la participación de la fuerza laboral, las tasas de cobertura y el grado de cumplimiento con las contribuciones en cada país.
} 
ra en América Latina) y examinar otras variables importantes del sistema, como las obligaciones por concepto de pensiones y la rentabilidad interna del sistema, que son sumamente difíciles o imposibles de calcular directamente sólo sobre la base de los datos existentes.

\section{Efectos del envejecimiento sobre el gasto y el balance del sistema}

El gráfico 3 ilustra las tendencias generales previstas de estas dos variables a medida que la población envejece. Las cifras presentadas corresponden a valores fijos de la tasa de cotización ( $\mathrm{c}=12.2 \%$ ), la participación de la masa salarial en el PIB $(\mathrm{s}=34.3 \%)$ y la proporción de trabajadores cubierta por el sistema de pensiones respecto al número de trabajadores asalariados $(\mathrm{k}=0.76)$, lo que corresponde al promedio de los países latinoamericanos alrededor de 1990. En el caso del balance financiero, se formula otro supuesto de que no existe un fondo de reserva o deuda constituidos, que puedan compensar los desequilibrios periódicos; en otras palabras, nuestra definición del balance refleja el déficit o superávit primario.

En el gráfico 3 se aísla el efecto demográfico de la manera estándar, fijando el valor de otros factores concomitantes y examinando la variación de los indicadores de interés dentro de un rango plausible de nuestra variable de envejecimiento, $d$. Se representan cuatro puntos de referencia para cada indicador del sistema. Los dos primeros puntos, de izquierda a derecha, se obtienen sobre la base del perfil demográfico (estructura según edades adultas y tasa de mortalidad) de Nicaragua en 1990, y del de Uruguay en 1990, países que poseen la estructura etaria más joven y la más vieja de la región, respectivamente. Además, a fin de tener una idea de la posible evolución futura a largo plazo de los indicadores del sistema de pensiones, el tercer punto corresponde al perfil demográfico de Francia en 1990, y el cuarto al perfil proyectado de Francia para el 2050 (Dinh, 1995; Meslé y Vallin, 1998).

La sección A del gráfico refleja la relación lineal del gasto del sistema de pensiones con el envejecimiento (indizado por $d$ ), ceteris paribus, incorporado en las condiciones del modelo. Los valores de los datos confirman el hecho, ya documentado en la sección II del artículo, que cuando los sistemas cubren una fracción de una población en edad de trabajar demográficamente "joven", el gasto en pensiones equivale a sólo $1 \%$ o $2 \%$ del PIB, pero que puede llegar a niveles cercanos
GRAFICO 3

\section{Simulación del gasto y el balance financiero del sistema de pensiones en función del envejecimiento, según las condiciones del modelo a}

A. Envejecimiento y gasto en pensiones

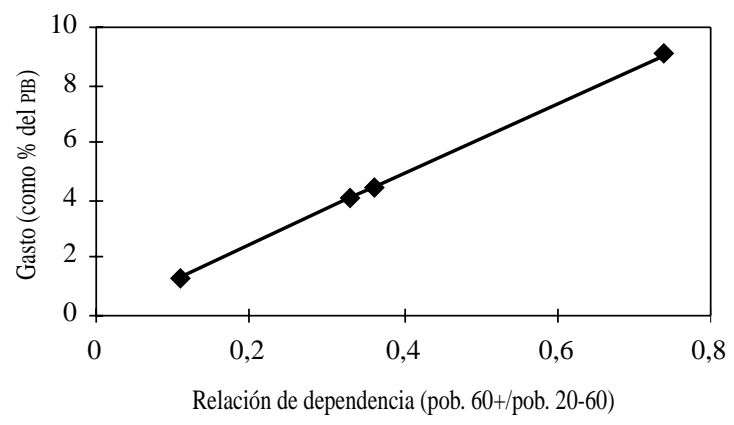

B. Envejecimiento y balance del sistema de pensiones (\% del ingreso)

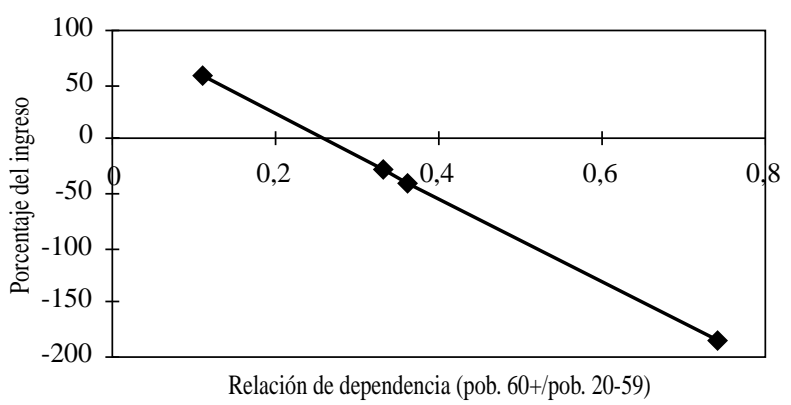

C. Envejecimiento y balance del sistema de pensiones (\% del PIB)

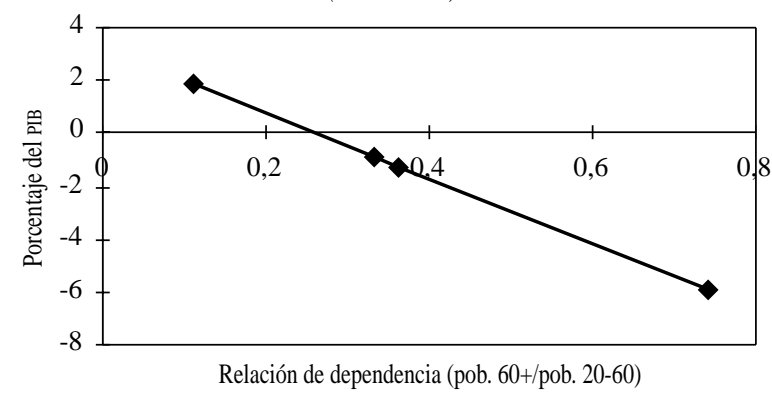

a Según lo descrito en la sección III, apartado 1. Los supuestos y las ecuaciones del modelo figuran en el apéndice 2. En esta simulación se utilizan parámetros "típicos", es decir, promedios de los sistemas de pensiones y el mercado laboral latinoamericanos, mientras que se permite que varíen las condiciones demográficas dentro de la gama señalada. De izquierda a derecha, los puntos reflejan los perfiles demográficos de Nicaragua en 1990, de Uruguay en 1990, de Francia en 1990, y de Francia en 2050, respectivamente. 
al $10 \%$ cuando la población nacional se torna muy envejecida, incluso manteniendo la cobertura muy incompleta (alrededor de la mitad de la fuerza de trabajo) que América Latina en su conjunto tiene actualmente. Los niveles de gasto pueden ser más elevados en la práctica, cuando la cobertura es más amplia o universal: el gasto en pensiones ya alcanza a un $15 \%$ del PIB en Italia actualmente y, como ya se señaló, se proyecta que se incremente incluso más en algunos países de la OCDE durante el siglo XXI. ${ }^{13}$

Mientras los niveles de gasto dan una indicación del peso que los programas de pensiones tienen en la economía, el desempeño financiero agregado del sistema se caracteriza mejor mediante el balance, es decir, la diferencia entre los ingresos anuales del sistema provenientes de las cotizaciones y el pago anual de pensiones. Las secciones $\mathrm{B}$ y $\mathrm{C}$ del gráfico 3 muestran dos posibles variantes de este indicador: el balance como fracción de los ingresos del sistema y como porcentaje del PIB. Ambas son también funciones lineales de $d$, según las condiciones del modelo. Estas líneas sugieren que, si no se ajustan las variables del sistema (tasas de cotización, tasas de reemplazo y condiciones de afiliación), el envejecimiento demográfico puede transformar el superávit del balance inicial cuando las poblaciones son "jóvenes" ( $d$ menor que 0.3$)$ en déficit considerables, hasta del orden de dos veces los ingresos del sistema, o de un $6 \%$ del PIB, cuando las poblaciones se vuelven muy envejecidas ( $d$ alrededor de 0.8) bajo parámetros promedios constantes de los sistemas latinoamericanos. Esta posibilidad teórica, sin embargo, no se ha verificado hasta ahora en la región. En los sistemas más maduros y "envejecidos" de los países más desarrollados, las variables del sistema (cotizaciones, monto de los beneficios o condiciones de elegibilidad) normalmente se ajustan antes de que ocurran tales situaciones extremas.

\section{Sustentabilidad de los sistemas de reparto y equidad intergeneracional}

No siempre es fácil definir qué es un nivel "sustentable" de gasto en pensiones o un nivel "insustentable" de déficit, ya que en la práctica ello involucra muchas variables concomitantes. En los debates públicos, las

\footnotetext{
${ }^{13}$ Una simulación con parámetros típicos de los sistemas de pensiones latinoamericanos, basada en el presente modelo (Bravo, 1999), indica que en condiciones de cobertura universal los niveles de gasto en la región duplicarían los presentados en la sección A del gráfico 3 .
}

consideraciones políticas pueden llevar a describir como excesivo un déficit corriente o proyectado relativamente moderado al tratar de justificar reformas que entrañan un cambio de régimen, mientras que déficit considerables quizás no sean considerados un problema grave si pueden financiarse con un incremento de la deuda pública, la que deberán encarar las futuras administraciones y pagar las generaciones futuras de contribuyentes.

Pero incluso dejando de lado las consideraciones políticas para fines analíticos, la definición de sustentabilidad sigue siendo compleja, especialmente cuando se limita a los índices estándares del sistema periódico. Por ejemplo, un nivel de gasto en pensiones extraordinariamente elevado (como en Italia, por ejemplo) refleja sobre todo el hecho de que su población es actualmente una de las más envejecidas del mundo. Resulta muy natural que, tanto a nivel individual como societal, los recursos destinados a financiar el consumo de las personas de edad aumenten a medida que la duración de la vida se extiende a edades cada vez más avanzadas. En sí misma, ésta no puede considerarse una tendencia "insustentable"; la insolvencia sólo se produce si no hay una provisión adecuada para financiar ese mayor gasto durante un período de tiempo razonablemente prolongado, consistente con el horizonte temporal durante el cual el sistema de pensiones contrae obligaciones y paga beneficios. ${ }^{14}$

El balance financiero (del período) da más información al respecto, pero tampoco ofrece una prueba clara de solvencia, puesto que los desequilibrios financieros primarios pueden ocurrir simplemente como resultado de los diferentes grados de maduración de los sistemas o de fluctuaciones económicas temporales, que no implican insolvencia en el sentido que acaba de señalarse. Los déficit abultados y persistentes (como los proyectados para varios países de la OCDE durante el siglo XXI, en que todas las variables permanecen constantes excepto el envejecimiento) requieren obviamente de ajustes, pero incluso en este caso debe establecerse una distinción clara entre la "sustentabilidad" de los valores de los parámetros (nivel de cotizaciones, modo de cálculo de los beneficios, condiciones de elegibilidad), que pueden modificarse, y la viabilidad del diseño general del sistema, que tiene más que ver con su consistencia con un tipo determinado de mo-

\footnotetext{
14 Baldacci y Tuzi (1999) brindan un análisis interesante de los cambios demográficos y del mercado laboral, y la sustentabilidad del sistema de pensiones italiano.
} 
delo de desarrollo y es más una función de consideraciones de economía política que del grado de envejecimiento de la población per se.

Los análisis actuariales completos que toman en cuenta los parámetros presentes y futuros son un medio más satisfactorio de evaluar la solvencia y la sustentabilidad a mediano y largo plazo de los sistemas. De hecho, en un mundo de certidumbre acerca de los valores corrientes y la evolución futura de las diferentes variables del sistema, el balance actuarial sería una excelente medida resumen de la solvencia (a mediano plazo). Muchos sistemas de países desarrollados cuentan con una práctica de evaluación actuarial bien establecida. Lamentablemente, en la mayoría de los países latinoamericanos no se realizan estos análisis de manera consistente (véase Mesa-Lago, 1985 y 1991), aunque hacerlos contribuiría mucho a establecer una base más sólida para las discusiones de política (Mesa-Lago, 2000). No obstante, en cualquier caso específico estas evaluaciones deben interpretarse con precaución, dada la incertidumbre acerca del futuro, sobre todo a mediano y largo plazo, pues los resultados dependen directamente de la validez de los supuestos formulados.

En este contexto, los análisis de política utilizan cada vez más indicadores que permitan examinar la situación financiera comparativa a mediano y largo plazo de los sistemas, ${ }^{15}$ que se analizan con más detalle en la sección siguiente. Uno de estos indicadores es la deuda (u obligación) pensional implícita, es decir, el valor presente de las obligaciones del sistema de reparto con los jubilados y con los trabajadores activos en un momento determinado. Este indicador, calculado según las condiciones del modelo, se muestra en la sección A del gráfico 4, en función de nuestro índice preferido de envejecimiento $d$, apreciándose que aumenta casi linealmente respecto de la relación de dependencia dentro del rango señalado.

La deuda pensional implícita es un indicador resumen útil y, como se verá más adelante, resulta clave para conocer y medir el costo fiscal de terminar un sistema de reparto existente. Pero no nos dice directamente si un sistema dado es sustentable o no. Tal como en el caso del gasto, la obligación pensional aumenta naturalmente a medida que el sistema madura y expande su cobertura, y la población envejece. Asimismo, si por ejemplo una deuda implícita considerable puede seguir

15 Véase un buen análisis crítico de los diferentes indicadores en Franco, 1995.
GRAFICO 4

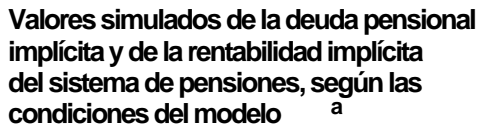

A. Deuda pensional implícita y envejecimiento

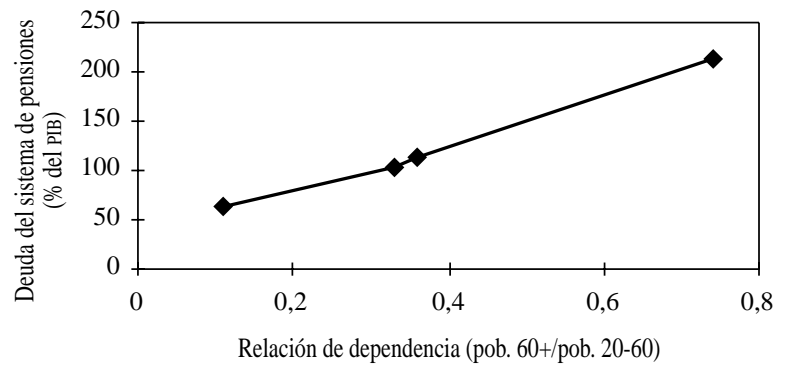

B. Rentabilidad del sistema de pensiones y envejecimiento

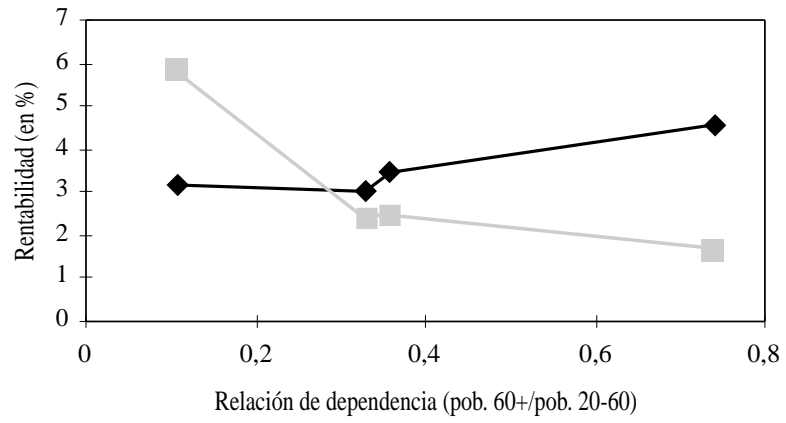

a Según lo descrito en la sección III apartado 1. Los supuestos y las ecuaciones del modelo figuran en el apéndice 2. En esta simulación se utilizan parámetros "típicos", es decir, promedios de los sistemas de pensiones y del mercado laboral latinoamericano, mientras que se permite que varíen las condiciones demográficas dentro de la gama señalada. De izquierda a derecha, los puntos reflejan los perfiles demográficos de Nicaragua en 1990, de Uruguay en 1990, de Francia en 1990, y de Francia en 2050, respectivamente.

"traspasándose" a las generaciones futuras (como en un sistema de reparto continuo) en condiciones aceptables para los trabajadores y los jubilados, entonces no hay argumentos contundentes para considerarla inviable. ${ }^{16}$ Además, es posible que un sistema con dependencia baja tenga una deuda implícita pequeña $\mathrm{y}$, al mismo tiempo, un déficit permanente importante, como lo señalan las estimaciones del modelo para

\footnotetext{
${ }^{16}$ Desde una perspectiva macroeconómica, un criterio válido, especialmente para los países muy endeudados y con sistemas relativamente maduros, es el incremento de las obligaciones del sector público (que incluyen las del sistema de pensiones). En este sentido, Chand y Jaeger (1996) hacen un muy buen análisis de varios países industrializados.
} 
Guatemala y El Salvador (no mostradas aquí). De hecho, Nicaragua, un país con deuda pensional baja, tenía un déficit operacional modesto a comienzos de los años noventa. También puede darse la situación contraria: países con una alta deuda pensional, con una población relativamente envejecida, que muestran un equilibrio financiero sostenido o incluso un superávit presupuestario (por ejemplo, las estimaciones del modelo para Chile y Uruguay). Costa Rica, un país con una deuda relativamente elevada, tenía un superávit operacional a comienzos de los años noventa; un caso más extremo es el de los Estados Unidos, que exhibe una deuda pensional elevada, pero un superávit periódico muy sustancial. Varios otros países de la OCDE tienen obligaciones incluso más abultadas y operan con déficit modestos o con presupuestos equilibrados.

En ciertos casos, se puede obtener un diagnóstico más consistente cuando el gasto, el déficit financiero y la deuda son sustanciales, como por ejemplo en Argentina y Uruguay durante los años ochenta. Cuando todos los indicadores apuntan en la misma dirección, la necesidad de efectuar ajustes importantes al sistema se vuelve más obvia. Pero en muchos casos teóricos y situaciones del mundo real, los indicadores mencionados no permiten una evaluación inequívoca, lo que refuerza la idea de que no existe una norma de aplicación general para justificar la necesidad de un tipo particular de reforma exclusivamente sobre la base de las tendencias del envejecimiento. ${ }^{17}$

Otra forma de abordar el problema es preguntándose cómo afecta el sistema a los diferentes grupos generacionales. De hecho, un criterio de creciente aceptación para definir la sustentabilidad de la política pública o de un determinado programa de gobierno, como en nuestro caso actual, es considerar que un sistema o una política adolece de inequidad intergeneracional, si brinda beneficios a las generaciones actuales a expensas del bienestar económico de las generaciones futuras. En casos extremos, un determinado programa o política puede resultar del todo insostenible, si exige que las generaciones futuras soporten una carga tributaria neta excesivamente grande. Un ejemplo de esta última situación lo aporta Gokhale (1995), quien estima, sobre la base de cuentas generacionales para los Estados Unidos, que la continuación de las po-

17 Véase en Blanchet (1990 y 1998) una discusión detallada y matizada de los méritos relativos de los sistemas de reparto y de capitalización en condiciones demográficas cambiantes dentro de un marco de equilibrio general, que refuta varios conceptos erróneos al respecto. líticas actuales del gobierno exigiría que las generaciones futuras pagaran una carga tributaria neta $35 \%$ mayor que las generaciones existentes, lo que equivaldría a una cifra claramente inconcebible de $80 \%$ de su ingreso.

Siguiendo este enfoque, la sección B del gráfico 4 indica la rentabilidad implícita del sistema de pensiones $(\rho)$, que refleja el beneficio vitalicio neto de la participación en el sistema para un individuo o cohorte determinada. $\rho$ mide el valor presente que el individuo o cohorte en cuestión obtiene en beneficios pensionales en exceso del de las cotizaciones abonadas al sistema durante su vida laboral, un indicador análogo a la rentabilidad de las inversiones financieras. ${ }^{18}$ Tal como en el caso de las variables graficadas previamente, este indicador se calcula bajo los supuestos generales de nuestro modelo básico, considerando ahora dos escenarios alternativos. Primero, el caso en que las variables del sistema permanecen fijas durante todo el proceso de envejecimiento, y en que el presupuesto público general absorbe cualquier desequilibrio que pueda presentarse en el sistema de pensiones. Segundo, el caso en que la relación entre cotizaciones y beneficios está programada para ajustarse a los cambios del perfil demográfico de modo que se obtiene un equilibrio financiero en todo momento. Ninguna de estas dos funciones de $d$ es estrictamente lineal, por las razones que se explican a continuación.

La primera línea indica que, cuando la relación contribuciones/beneficios se mantiene constante, la mayor supervivencia en las edades adultas que se produce en el proceso de envejecimiento implica primero una ligera disminución, y luego un incremento importante de $\rho$. Así ocurre, porque los descensos más importantes en la mortalidad adulta se obtienen primero para las edades de "adulto joven" o laborales, mientras que los ulteriores se concentran más en edades más avanzadas (de jubilación). Esto lleva a que la relación entre los años vividos como jubilado y los años vividos como trabajador primero se estanque o disminuya ligeramente, y luego se eleve notoriamente.

La segunda línea muestra que si la relación contribuciones/beneficios se mantiene siempre a niveles de equilibrio financiero, entonces la rentabilidad del sis-

\footnotetext{
18 Para una discusión más detallada de la rentabilidad implícita y de cómo se ve afectada por diferentes factores y políticas, véase Bravo (1996). Los efectos conexos sobre la riqueza vitalicia se estudian en Auerbach y Kotlikoff (1987), Arrau (1991) y Cifuentes (1995), en el contexto de modelos de equilibrio general y de generaciones superpuestas.
} 
tema declina rápidamente con $d$, al menos hasta los niveles "moderados" de envejecimiento, y luego lo hace a un ritmo más lento. La explicación es la siguiente. Cuando la población envejece en un sistema equilibrado, se requiere incrementar las contribuciones o disminuir los beneficios. Dado que las primeras etapas del envejecimiento de la población adulta obedecen sobre todo a reducciones de la fecundidad - no a la supervivencia en edades avanzadas-, el ajuste de la relación contribuciones/beneficios es considerable en comparación con las mejoras modestas de la supervivencia en los adultos. Después de $d=0.8$, las reducciones de $\rho$ se vuelven mucho menores, puesto que gran parte del cambio en $d$ es producida por los incrementos de la relación entre los años vividos en edades avanzadas y los vividos en las edades de adulto joven, lo que tiene un efecto positivo sobre $\rho$ que compensa en buena parte el efecto negativo del aumento necesario en la relación contribuciones/beneficios.

¿Cuál de estos dos escenarios —el de contribución y tasa de reemplazo fijos o el régimen de equilibrio financiero- es más "sustentable", en el sentido de minimizar la inequidad intergeneracional? El gráfico sugiere que la mantención de reglas fijas en el sistema durante las primeras etapas del proceso de envejecimiento (hasta un $d=0.3$, en el gráfico 4 ) produce una rentabilidad razonablemente estable, mientras que en las etapas más avanzadas del envejecimiento la regla del equilibrio financiero daría diferencias menores de rentabilidad entre las cohortes. Interpretando los mismos datos con una perspectiva algo diferente, se desprende que en los inicios del proceso se otorgarían transferencias desproporcionadamente grandes a las cohortes iniciales, si el sistema se ajustara al equilibrio financiero, es decir, si todos los ingresos se transfirieran (mecánicamente) en forma de pensiones, sin tomar en cuenta las contribuciones previas de los pensionados. El gráfico ilustra también el riesgo de ofrecer beneficios demasiado generosos a las cohortes más tardías, pues el sistema entra en un desfinanciamiento crónico (después de $d=0.3$, en el gráfico) si la relación beneficios/contribuciones no se ajusta según las condiciones del contexto demográfico más envejecido.

Por tanto, en el proceso de envejecimiento de largo plazo, la siguiente secuencia de políticas podría evitar grandes diferencias en la rentabilidad obtenida por diferentes cohortes: i) fijar tanto las tasas de contribuciones como las de beneficios durante las etapas iniciales del proceso de envejecimiento, y ii) cuando el envejecimiento se halle más avanzado, cambiarse a la norma de equilibrio financiero, que aseguraría una mayor equidad intergeneracional en esa etapa. La primera parte de esta secuencia - ofrecer beneficios definidos a cambio de contribuciones más o menos determinadas - ha sido de hecho la regla seguida tradicionalmente por muchos países; la mayoría de los sistemas actuales siguen todavía definidos por beneficios. Sin embargo, como lo demuestra la experiencia más reciente, el cambio a la norma de la segunda etapa ("equilibrio financiero") ha demostrado ser más problemático, porque aunque las restricciones fiscales prácticamente obligan al cambio, éste entraña una pérdida (con respecto al escenario sin cambio) para las generaciones presentes de trabajadores, que a menudo es resistido políticamente. ${ }^{19} \mathrm{La}$ resistencia exitosa al cambio y al financiamiento directo o indirecto por las cohortes actuales de trabajadores significa, no obstante, transferir el déficit actual a las generaciones futuras y, de esta forma, vulnerar la equidad intergeneracional.

En este contexto, se pueden adoptar dos enfoques básicos para alcanzar el equilibrio financiero: el más común y tradicional, enmarcado en términos de una lógica y terminología de los beneficios definidos, implica cambios o reformas "paramétricos" en que se modifican las cotizaciones, se ajustan los beneficios, o se restringen más las condiciones de elegibilidad. El otro consiste en establecer explícitamente el doble objetivo de la solvencia financiera y la equidad intergeneracional, y especificar un diseño del sistema que pueda albergar a ambas. Uno de los enfoques de esta índole se conoce como esquema de "contribución nocional", que se ha aplicado en Suecia, Italia y Letonia, y está en estudio en otros países de Europa oriental (Holzmann, 1997; CEPAL, 1998). Se trata esencialmente de un esquema definido en función de las contribuciones, financiado principalmente bajo la modalidad de reparto, y que constituye pequeñas reservas para atenuar posibles desequilibrios transitorios. En estos esquemas se instituyen registros individuales (análogos a las "cuentas" de los sistemas de capitalización), que acumulan contribuciones más intereses, a una tasa dada por el crecimiento anual de la masa sa-

\footnotetext{
${ }^{19} \mathrm{Si}$ el superávit generado desde el inicio del sistema se invierte adecuadamente y se destina a cubrir los déficit en su etapa madura, entonces el paso a la regla de equilibrio puede facilitarse enormemente. Esta es de hecho una recomendación sensata que puede formularse a los países que tienen sistemas incipientes y poblaciones jóvenes, pero es un consejo que llega demasiado tarde para los que ya han gastado el superávit o que han dejado que el valor real de las reservas se anule por rentabilidades negativas.
} 
larial. Dentro del conjunto de regímenes de reparto, esta clase de sistema favorece la justicia actuarial, incrementa la probabilidad de un equilibrio financiero del sistema en el mediano y largo plazo, y modera las inequidades intergeneracionales. En la región, interesa señalar el caso de Brasil, el país que con mayor claridad está apuntando en esa dirección (Pinheiro y Vieira, 2000; Uthoff, 1999).

Desde la perspectiva demográfica, este tipo de sistema tiene la ventaja de ajustarse endógenamente a los cambios en la mortalidad de edad avanzada y de ofrecer una protección razonable contra las fluctuacio- nes de la estructura etaria o del mercado laboral. En un mundo incierto, los sistemas de reparto definidos en función de las contribuciones tienden a mejorar la distribución intergeneracional de riesgos y a incrementar el bienestar a lo largo del ciclo de vida (Thogersen, 1998). Por cierto que el sistema no es inmune a riesgos potenciales, ${ }^{20}$ pero estos parecen relativamente menores que los que afectan a los sistemas tradicionales definidos por beneficios o a los esquemas de capitalización plena. También resulta clave en estos esquemas el seguimiento actuarial apropiado, a fin de evitar desequilibrios por períodos de tiempo extensos. ${ }^{21}$

\section{IV}

\section{La deuda pensional implícita: concepto e indicadores}

Decíamos que el debate público de política en torno a los sistemas de pensiones está desplazando gradualmente su foco desde el desempeño financiero en el corto plazo (niveles actuales de gasto o de déficit/superávit), hacia la situación financiera en el mediano a largo plazo. Esto es especialmente válido en el caso de los países más desarrollados, donde el envejecimiento de la población se inició hace muchas décadas y el proceso está mucho más avanzado. En América Latina, varios análisis recientes han utilizado proyecciones de mediano plazo que incorporan las tendencias demográficas de modo más o menos detallado, por ejemplo, estudios para México, Colombia, Chile, Uruguay y Argentina (véase una revisión crítica de ellos en MesaLago, 2000) y para Brasil (Uthoff, 1999), en respuesta a inquietudes de política que se analizarán más adelante. Algunos de ellos examinan explícitamente la noción de la deuda pensional implícita.

Un tipo de indicador resumen utilizado en los estudios recientes es el pasivo del sistema de pensiones, una medida convencional que se refiere al valor presente de las obligaciones con los participantes en el sistema (Van der Noord y Herd, 1994; Franco, 1995; Holzmann, 1997; Vernière, 1997a). Este tipo de indicador se fundamenta en la idea de que existe un contrato implícito en los sistemas de reparto en virtud del cual el Estado, a cambio de los aportes de los trabajadores, se compromete a pagar las pensiones de las cohortes jubiladas con las contribuciones recaudadas de la ge- neración (en edad de trabajar) siguiente. De esta manera, resulta lógico estimar el valor descontado (presente) de los flujos de ingresos y pagos para un determinado momento o período de tiempo y número de cohortes. En los países más desarrollados y envejecidos, el estudio de estas obligaciones emana del interés general en evaluar la situación financiera del gobierno en el mediano y largo plazo (que incluye los niveles de gasto, déficit y deuda pública) dado el envejecimiento actual y proyectado de la población, que se ve afectada por las tendencias de largo plazo y por el "eco" inminente del auge de nacimientos que se produjo hace unas cuatro décadas en esos países.

Algunos observadores interpretan que la solicitud de Gran Bretaña a la Comisión Europea de incorporar los pasivos de los sistemas de reparto de la seguridad social, entre los criterios para pasar a la moneda única, reflejó la inquietud de que, dado que las obligaciones del sistema público en el Reino Unido son menores que en los demás países de la Unión Europea, existía el riesgo de que los contribuyentes británi-

\footnotetext{
${ }^{20}$ Relacionado sobre todo con el riesgo de rendimientos bajos o negativos de la inversión de reservas (relativamente exiguas) y de la presión política contingente para actuar conforme a la fórmula de las pensiones definidas por beneficios, susceptibles de brindar una tasa de reemplazo salarial baja, especialmente durante los episodios de elevado crecimiento salarial.

${ }^{21}$ Véase un análisis de las características y algunos de los problemas que pueden surgir en estos esquemas en Valdés-Prieto (1999).
} 
cos tuvieran que financiar los pasivos de otros países o ser sancionados con tasas de interés más elevadas para costear esas obligaciones (Vernière, 1997a, p. 2). En los Estados Unidos, se hace más hincapié en la posibilidad de afrontar, sin graves consecuencias fiscales y de distribución intergeneracional, el gasto que deberá hacer el gobierno (incluyendo pensiones y programas de salud pública) cuando jubilen las generaciones del "baby boom". En esta línea cabe interpretar que las obligaciones implícitas y el costo de liquidarlas hayan sido una consideración importante en las propuestas de reforma de la seguridad social debatidas con ocasión de la campaña presidencial estadounidense del 2000. En América Latina, el interés también es relativamente reciente y, siendo distinto el contexto demográfico, no está motivado tanto por la inquietud sobre el envejecimiento de la población, sino por la carga fiscal que este tipo de gasto puede representar hoy y en el futuro cercano, en contextos de restricciones y reformas fiscales. Este es el tema que se tratará a continuación.

\section{Definición y medición}

Hay diversas denominaciones y especificaciones de la deuda (obligación) pensional implícita que tienen significados y usos ligeramente distintos (Holzmann, 1997; Van der Noord y Herd, 1994). Las definiciones principales son tres, a saber: i) obligaciones devengadas a la fecha, que representan el valor presente de las pensiones por pagar considerando sólo los derechos devengados por los trabajadores y jubilados en un determinado momento, excluyendo las contribuciones futuras y la devengación futura de derechos; ii) obligaciones del sistema cerrado, que mide las obligaciones con todos los pensionados y trabajadores actuales, incluidas sus contribuciones futuras y su devengación futura de derechos; y iii) obligaciones del sistema abierto, que incluyen también las contribuciones y derechos que adquirirán los futuros integrantes de la fuerza de trabajo.

La definición más pertinente para calcular los costos de la transición del sistema de reparto al de capitalización corresponde a una del primer tipo. El uso de cualquiera de las variantes depende de cuál es el escenario de la reforma que se considere: si el cambio a la capitalización es parcial o total, si es voluntario u obligatorio para diferentes generaciones de trabajadores (actuales y futuras), si es inmediato o gradual, etc. Para el análisis siguiente, basado en gran medida en los trabajos realizados por el autor en colaboración con Andras Uthoff (Uthoff y Bravo, 1998; Bravo y Uthoff, 1999), empleamos una definición que considera el flujo futuro de pensiones devengadas por los jubilados actuales, más una compensación de las contribuciones previas a todos los que integran actualmente la fuerza laboral. Esta definición difiere un poco de la especificación estándar "devengada a la fecha": sólo el primer componente es distinto, dado que considera el reembolso de las contribuciones previas en vez de los derechos pensionales. Las dos medidas deberían tener el mismo orden de magnitud, pero puede que no coincidan exactamente en todos los casos.

El contexto de política es en forma muy somera el siguiente: sabemos que en América Latina las reformas recientes incluyen el ajuste de los requisitos y beneficios, y en varios casos, la incorporación de al menos un componente de capitalización de las contribuciones y beneficios. Entre estos casos están la reforma temprana de Chile de 1981, la que, junto con las de México (1995), Bolivia (1996) y El Salvador (1997), dictaron un viraje total desde sistemas estatales de reparto definidos por beneficios a esquemas de gestión privada, de capitalización total, definidos por contribuciones individuales. Otras reformas han mantenido un componente de reparto estableciendo sistemas mixtos (multipilar) o paralelos, como las de Perú (1992), Argentina (1993), Colombia (1993) y Uruguay (1995).

Se supone que el componente de capitalización debería aliviar la carga financiera que imponen los sistemas de reparto al presupuesto fiscal en el contexto de las tendencias demográficas y del mercado de trabajo mencionadas, y así estimular una mayor eficiencia en la gestión del sistema, conducir a niveles más altos de ahorro e inversión nacionales, y promover el desarrollo de los mercados financieros donde se invierten los fondos de pensiones. La evidencia es ambigua en lo que respecta a estos presuntos bienes, en parte, porque en muchos países con reformas recientes no ha transcurrido todavía un tiempo suficiente para hacer una buena evaluación. Pero aún en el caso temprano de Chile, donde son evidentes el crecimiento y desarrollo de los mercados financieros y de su marco regulatorio, no está claro si la capitalización ha traído consigo aumentos importantes del ahorro nacional o de la inversión productiva (Uthoff, 1997), o si ha contribuido a reducir los costos de administración, que todavía representan más del $20 \%$ de las cotizaciones. ${ }^{22}$

\footnotetext{
${ }^{22}$ Los datos recientes (presentados en Mesa-Lago, 2000, cuadro 6) implican que los costos de administración varían considerablemente desde un $4 \%$ de las contribuciones en Bolivia a más de $30 \%$ en Argentina. Véase Valdés-Prieto (1999) para un análisis más detallado de los costos y las dificultades de hacer comparaciones estrictas entre países y empresas.
} 
Aunque existen varios modelos que pronostican o sugieren aumentos del ingreso nacional y del bienestar en el largo plazo con el cambio a sistemas de capitalización (véanse Schmidt-Hebbel, 1997; Valdés-Prieto, ed., 1997, y las referencias que allí se incluyen), parece algo prematuro evaluar este efecto, potencialmente importante, en la mayoría de los países que han introducido reformas en este sentido.

Además, lo que es de interés específico para este estudio, la transición de sistemas de reparto a sistemas de capitalización no produce necesariamente un alivio de la carga fiscal en el corto y mediano plazo; por el contrario, un análisis reciente de la CEPAL (1998) y este mismo sugieren que en realidad esa transición genera grandes obligaciones fiscales, que deben ser pagadas por las actuales y futuras generaciones de trabajadores. Estos costos se producen, porque la transición obliga a hacer explícita y a pagar parte o toda la deuda pensional implícita, sin que se reciban como ingreso las contribuciones efectuadas por los que se cambian al nuevo sistema.

En las secciones siguientes, buscamos medir la cuantía de la deuda pensional implícita y derivar los costos fiscales asociados mediante el uso de un modelo simple, y de la información disponible sobre las variables demográficas, del mercado de trabajo y del sistema de pensiones, y analizamos los factores y resultados para los países latinoamericanos considerados.

\section{Obligación que debe hacerse explícita al cam- biar de régimen}

El escenario base previsto es el de una sustitución completa de un sistema de reparto estatal por un sistema de capitalización total con gestión privada. En este caso, los pasivos ("deuda" del gobierno por concepto de pensiones), calculados a valores actuariales justos, corresponden al valor presente de las pensiones devengadas y por devengarse en años futuros de todos los pensionados del sistema preexistente de reparto, más el valor presente de las contribuciones de los trabajadores afiliados activos al momento de la reforma. ${ }^{23}$ Para medir directa y exactamente estas obligaciones, sería necesario tener series cronológicas completas y detalladas de los perfiles de edad de participación en la fuerza de trabajo, empleo, tasas de cobertura y cum-

\footnotetext{
${ }^{23}$ Una definición natural alternativa de este último componente, la de los derechos de pensiones devengados a la fecha por los activos al momento de la reforma, es teóricamente menos precisa y empíricamente menos abordable.
}

plimiento, y los ingresos del trabajo para - digamoslos últimos 40 años. Como esta base de datos ideal no existe en país alguno de América Latina, desarrollamos un modelo que puede entregar una estimación razonable utilizando los datos demográficos, macroeconómicos y del mercado del trabajo de amplia disponibilidad.

Para nuestros cálculos utilizamos diversos supuestos, algunos altamente simplificadores, los más importantes de los cuales son: i) existe un esquema de pensiones de reparto desde 1950; ii) la tasa de cobertura global permanece constante al nivel de 1985 (momento intermedio entre 1950 y 2020, fin de nuestro período de proyección); iii) las tasas de contribución y reemplazo corresponden a las de principios de 1980 (Mesa-Lago, 1991); y iv) las contribuciones de todas las cohortes relevantes comienzan a la edad de 20 años y continúan sin interrupciones hasta su jubilación a los 60 años de edad. El conjunto completo de supuestos, así como la derivación de las ecuaciones que siguen, figuran en Bravo y Uthoff (1999). Usando este marco simplificado, es posible obtener estimaciones de la deuda pensional para un número relativamente grande de países latinoamericanos.

Bajo estos supuestos, las obligaciones previsionales con los económicamente activos $(D a)$ se pueden expresar, como fracción del PIB, de la siguiente manera:

$$
D a=c \cdot s \cdot k \cdot A a
$$

donde $c=$ tasa de contribución (expresada como porcentaje de los ingresos del trabajo); $s=$ participación de la masa salarial en el PIB; $k=$ cociente de número de trabajadores adscritos (cubiertos) al sistema entre el total de asalariados; $A a=$ promedio actualizado de años de cotización de la población activa.

De la misma forma, la deuda con los ya retirados (Dr) se expresa así:

$$
D r=r \cdot s \cdot k \cdot d \cdot A r
$$

donde $r=$ tasa de reemplazo (porcentaje de los salarios); $\mathrm{d}=$ relación de dependencia del sistema de reparto (población en edad de jubilación/población en edad de trabajar); $A r=$ promedio actualizado de años esperados de retiro por persona actualmente retirada, y las demás variables como ya fueron definidas. Tanto $A a$ como $A r$ son promedios actualizados ponderados, donde las ponderaciones están dadas por la población de los grupos de edad pertinentes. 
Las estimaciones basadas en este modelo se presentan en el gráfico 5; son válidas para nuestro caso especial base, en el que la tasa de descuento se asume igual a la de crecimiento de los salarios. ${ }^{24}$ Este gráfico muestra que la deuda pensional implícita es significativa en la mayoría de los países latinoamericanos, aun en algunos con población joven y baja cobertura de los sistemas, como la República Dominicana, Ecuador y Honduras. La deuda de pensiones es sustancial (más de $20 \%$ del PIB) en la mayoría de los países y extremadamente elevada (más de $200 \%$ del PIB) en aquellos de población más envejecida o de sistemas de alta cobertura como Argentina, Uruguay y Brasil.

En comparación, el nivel de las obligaciones pensionales de los países más desarrollados y envejecidos es en promedio mayor (del orden de $170 \%$ del PIB) que los de la región latinoamericana, pero varía menos entre los países, desde un mínimo estimado de $113 \%$ en los Estados Unidos a un máximo de $242 \%$ en Italia (OCDE, 1993).

Es interesante señalar que en la mayoría de los países con información disponible, la deuda pensional implícita es mayor - a menudo varias veces mayorque la deuda pública oficial. Según nuestras estimaciones, la deuda de pensiones equivale a un tercio de la deuda pública oficial de Ecuador, es entre una y dos

GRAFICO 5

América Latina y el Caribe:

Deuda implícita de pensiones

(Como porcentaje del PIB)

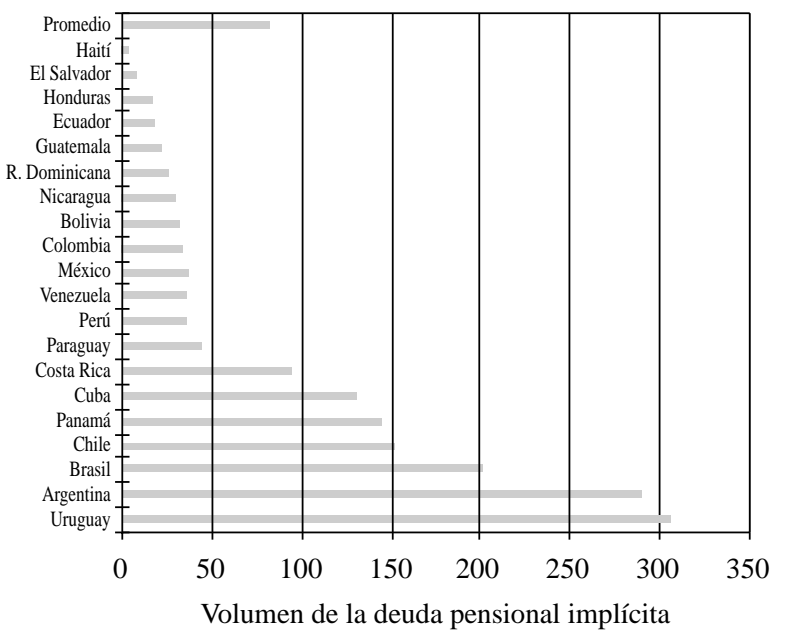

Fuente: CEPAL (1998), para el caso base en que la tasa de actualización es igual a la tasa de crecimiento salarial.

${ }^{24}$ Las consecuencias de levantar este supuesto se tratan en la sección V. veces la deuda pública de Perú, Venezuela y Colombia, más de cuatro veces la deuda pública oficial de Brasil, y entre 8 y 11 veces la deuda pública de Uruguay, Argentina y Chile. Estas últimas proporciones parecen también muy abultadas comparadas con las de los países más desarrollados y envejecidos; por ejemplo, la deuda pensional implícita de Italia, Francia, Alemania y el Reino Unido varía entre dos y cuatro veces su respectiva deuda pública oficial. ${ }^{25}$

Aunque varios de los supuestos del modelo son burdos y tienen sólo la validez de una primera aproximación, cabe destacar que nuestras estimaciones, cuando se calculan con los mismos datos básicos y valores de los parámetros, resultan razonablemente cercanos a otras estimaciones basadas en procedimientos alternativos. Por ejemplo, Schmidt-Hebbel (1995), utilizando un método distinto (el valor presente de los déficit proyectados de la reforma) y asumiendo otros parámetros, estima la deuda pensional colombiana en un rango entre $59.2 \%$ y $88.1 \%$ del PIB, mientras nuestra estimación (basada en parámetros prerreforma) llega sólo a $34.8 \%$. Sin embargo, utilizando los mismos parámetros del estudio citado, llegamos a un valor estimado de $62.5 \%$, que cae dentro de su rango de estimación. Nuestra estimación de la deuda pensional de Chile, $131 \%$ del PIB, no está lejos del valor de 126\% informado en Schmidt-Hebbel (1995, cuadro 3.8). Nuestra estimación para Brasil, de 201.6\% del PIB, está dentro del rango de estimación de otros estudios, que va desde $188 \%$ a $255 \%$ del PIB, dependiendo de los supuestos adoptados (Brasil, Gobierno, 1998). Como ejemplo final, el cálculo basado en nuestro modelo para Francia dio una deuda implícita de $224 \%$, cercana a la estimación de la OCDE, de $216 \%$ del PIB. Mesa-Lago (2000) se refiere a otras estimaciones, calculadas con métodos diferentes, señalando que no son estrictamente comparables con las que aquí se presentan.

\section{Efecto de la estructura etaria y de los patrones de mortalidad}

Las variables demográficas (básicamente la estructura por edad de la población y la mortalidad a edad avanzada) afectan el tamaño de la deuda, aunque se mostrará que su impacto no es tan grande como el de otras

\footnotetext{
${ }^{25}$ Las cifras de la deuda pública (como porcentaje del PIB) para los países latinoamericanos son de alrededor de 1995 (El Mercurio, 1997). Las estimaciones de la deuda pensional para los países de la OCDE son los derechos devengados hasta la fecha; las fuentes de datos son Van der Noord y Herd (1993) y, para la deuda pública como porcentaje del PIB, Le monde-Economie (1999, p. V)
} 
variables del sistema de pensiones y del mercado laboral. La estructura por edades de la población está representada directamente en la tasa de dependencia del sistema $(d=$ relación entre población de edad avanzada y población en edad de trabajar), pero entra también en el cálculo del número medio de años de cotización de la población activa $(A a)$ y en el del número esperado de años de jubilación de las personas actualmente retiradas $(A r)$. En este último caso, las condiciones de mortalidad en las edades avanzadas determinan el número esperado de años de vida que le queda a cada una de las cohortes que están actualmente en edad de jubilación. ${ }^{26}$

Para ilustrar la magnitud de los efectos demográficos sustituimos los valores medios de los países latinoamericanos analizados por valores nacionales extremos de envejecimiento y mortalidad. Más específicamente, primero construimos un vector de referencia compuesto por un conjunto de indicadores, definido como el promedio de cada una de las variables y estimamos un tamaño de la deuda de referencia. Luego recalculamos el valor de la deuda utilizando el nivel más alto y el más bajo de mortalidad en las edades mayores (Bolivia y Costa Rica, respectivamente) y la estructura de edad más joven y la más envejecida de la región (Nicaragua y Uruguay, respectivamente).

En el gráfico 6 se presentan los resultados. Ellos muestran que la mortalidad de edad avanzada tiene muy poca incidencia en el tamaño de la deuda: al sustituir el padrón de mortalidad "promedio" por el alto nivel de mortalidad de Bolivia, se obtiene una deuda pensional sólo algunos puntos porcentuales más baja. Algo similar sucede si se utiliza la baja mortalidad en edad avanzada de Costa Rica. Esto se debe principalmente a que, dentro de la región, la mortalidad en las

\footnotetext{
${ }^{26}$ Por construcción, el valor de $d$ es mayor mientras más vieja es la población y más baja es la mortalidad. El valor de $A a$ es más alto en poblaciones más envejecidas, ya que hay relativamente más personas con más años de contribución. Ar puede ser mayor o menor en poblaciones más envejecidas y de mortalidad más reducida: por una parte, una mortalidad más baja implica una mayor esperanza de vida para la mayoría de los tramos de edad pero, por otra, una estructura de población más vieja significa que los jubilados más jóvenes (de mayor esperanza de vida) tienen menor ponderación relativa en la población. Por lo tanto, el valor resultante de $A r$, así como el efecto "demográfico" final en el volumen de la deuda, depende de la particular combinación de estructura de edad y mortalidad de edad avanzada en cada país. Como se muestra en Bravo y Uthoff (1999), el efecto positivo del envejecimiento (que opera a través de $A a$ y $d$ ) tiende a dominar sobre la interacción ambigua entre mortalidad en las edades mayores y envejecimiento de la población, que opera a través de $A r$.
}

edades mayores no varía tanto en términos absolutos (que es lo que cuenta para el cálculo de la deuda pensional) como lo hace la mortalidad general. A comienzos de los años noventa, la esperanza de vida al nacer en América Latina era de 68.6 años, en Costa Rica 76.3 y en Bolivia 59.3 (una diferencia entre el mínimo y el máximo de alrededor de 17 años), mientras que la esperanza de vida a los 60 años era de alrededor de 18.9 para América Latina, 19.5 para Costa Rica y 15.2 para Bolivia, una diferencia entre el mínimo y el máximo de tan solo 4.3 años. Recuérdese, también, que la mortalidad en las edades mayores sólo afecta a la parte de la deuda con los jubilados, que es el menor de los dos componentes del total de la deuda pensional de casi todos los países latinoamericanos.

La estructura por edades de la población tiene un papel bastante más significativo: si se utiliza la distribución por edades de la población de Nicaragua, en vez de la del conjunto de América Latina, se obtiene una deuda pensional más de 9 puntos porcentuales inferior, y si se utiliza la estructura más envejecida de Uruguay, la deuda sube en más de 40 puntos porcentuales. Una comparación algo más realista es la que representan las dos últimas barras del gráfico 6 , donde se contrasta la configuración demográfica completa (distribución por edad y mortalidad de edad avanzada) de Nicaragua y Uruguay, dando un resultado casi idéntico al anterior: una diferencia en el tamaño de la deuda de casi $40 \%$.

Es importante destacar que los cambios en otros factores del sistema de pensiones y del mercado de trabajo pueden afectar en forma incluso más fuerte el volumen de la deuda: si Nicaragua tuviera la estructura de edad de Argentina, su deuda implícita aumenta-

GRAFICO 6

\section{Deuda pensional en diferentes condiciones demográficas}

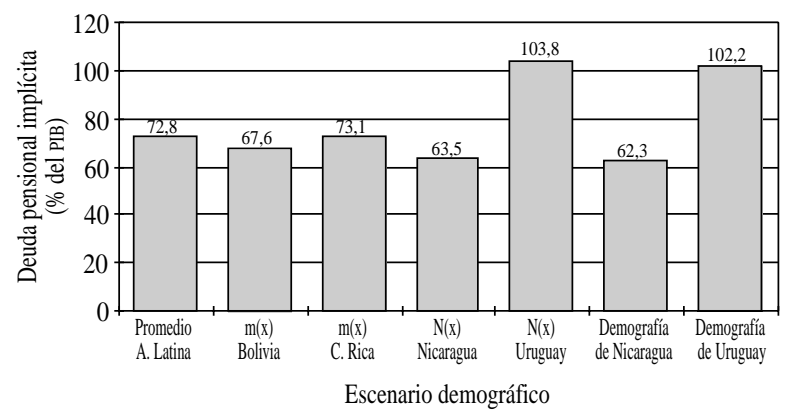

Fuente: Bravo y Uthoff (1999).

a $N(x)$ representa la distribución etaria de la población y $m(x)$ la mortalidad por edades. 
ría en más de la mitad de su valor actual, pero casi se duplicaría si tuviera la cobertura de población del sistema argentino, y más que se cuadruplicaría si tuviera la tasa de contribución de su sistema de seguridad social (Uthoff y Bravo, 1998).

El efecto demográfico potencial, por otro lado, pesa más en la determinación de la deuda que algunas variables de política macroeconómica. Una de ellas es la tasa de actualización que utiliza el gobierno para expresar las contribuciones pasadas y los beneficios futuros en valores presentes comunes. Para que esta tasa de descuento tenga un efecto comparable al de la estructura de edad, debería ser $2 \%$ más alta que la tasa de crecimiento salarial.
$\mathrm{Al}$ evaluar las obligaciones fiscales a mediano plazo, conviene proyectar la deuda implícita a alguna fecha futura — por ejemplo, como hizo Vernière (1997b) para Francia. La estimación correspondiente del efecto longitudinal del envejecimiento en este contexto es bastante robusta, puesto que, aunque sujetos a cierta incertidumbre, los cambios demográficos a mediano plazo son más predecibles, por ejemplo, que las tasas de interés o las tasas futuras de crecimiento económico. En particular, dado que la evolución de la relación de dependencia dentro de 20 a 25 años más estará determinada casi por completo por los patrones de mortalidad, que cambian gradualmente en el tiempo, la confianza en la tendencia proyectada es bastante alta.

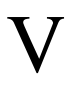

\section{Costos fiscales de transición}

El grado en que la deuda pensional de un país determinado es "manejable" o no, puede apreciarse mejor examinando el gasto fiscal anual que ella origina. Este costo fiscal depende del tamaño de la deuda y también de otras variables macroeconómicas y de política claves, como la tasa de crecimiento del PIB, la tasa de interés pagada por la deuda pública, los mecanismos de financiamiento y otros aspectos específicos de la reforma.

El gráfico 7 ilustra la magnitud del costo fiscal anual que se requeriría para pagar toda la deuda pensional implícita durante un período de 40 años (que es aproximadamente el período que se necesita para completar la transición), con una tasa anual de crecimiento del PIB de un $4 \%$ y diversas hipótesis respecto de $\triangle$ ), la diferencia entre la tasa de descuento $(i)$ y la tasa de crecimiento de los salarios $(\sigma)$.

La lectura de este gráfico es similar a la del gráfico 6: los costos fiscales anuales resultan bajos a "manejables" (menos de 1.5\% del PIB por año) en los grupos de países de deuda "baja" y "muy baja"; son bastante considerables — desde $1.4 \%$ hasta $6.7 \%$ del PIB- en el grupo de deuda "alta" (Cuba, Panamá, Chile, Costa Rica), y parecen demasiado altos en Argentina y Uruguay: estos dos países deberían hacer un esfuerzo financiero equivalente a entre $6 \%$ y $13 \%$ del PIB (dependiendo de los supuestos) por un período de 40 años, para cancelar totalmente los costos de transición del sistema de reparto al de capitalización. Las estimaciones mencionadas son más bien conservadoras, puesto que el supuesto estándar de eficiencia dinámica en los modelos de crecimiento implica una tasa de interés mayor que la masa salarial, por lo tanto $i$ es sustancialmente mayor que la tasa de crecimiento salarial. Nótese también que los costos fiscales (como porcentaje del PIB) no varían significativamente con el nivel de crecimiento del PIB; lo que más importa es su diferencia con la tasa de descuento (o de interés): una tasa de descuento $2 \%$ más alta que el crecimiento del PIB duplica con creces, en muchos casos, los costos fiscales en comparación con el caso en que $i=\sigma$.

Estas cifras medias anuales para un período de más de 40 años brindan algunos órdenes de magnitud útiles, aunque en las transiciones reales los costos fiscales varían a través del tiempo, dependiendo de las estipulaciones relativas al pago de la deuda pensional y los mecanismos de financiamiento. ${ }^{27}$ En particular, si se prolonga el período para pagar la deuda (por ejemplo, determinando una compensación periódica como en Argentina y Bolivia, en vez del "bono" pagadero a

\footnotetext{
${ }^{27}$ Dos mecanismos principales de financiamiento son los impuestos (corrientes) y la emisión de nueva deuda pública oficial (impuestos futuros). La mezcla de financiamiento afecta tanto el programa de amortización como la distribución de los costos de transición entre generaciones presentes y futuras de contribuyentes. Los efectos de distribución intergeneracional son importantes, pero no se analizarán en detalle aquí. Véanse Arrau (1991), Schmidt-Hebbel (1995), Cifuentes (1995) y Valdés-Prieto, ed. (1997).
} 


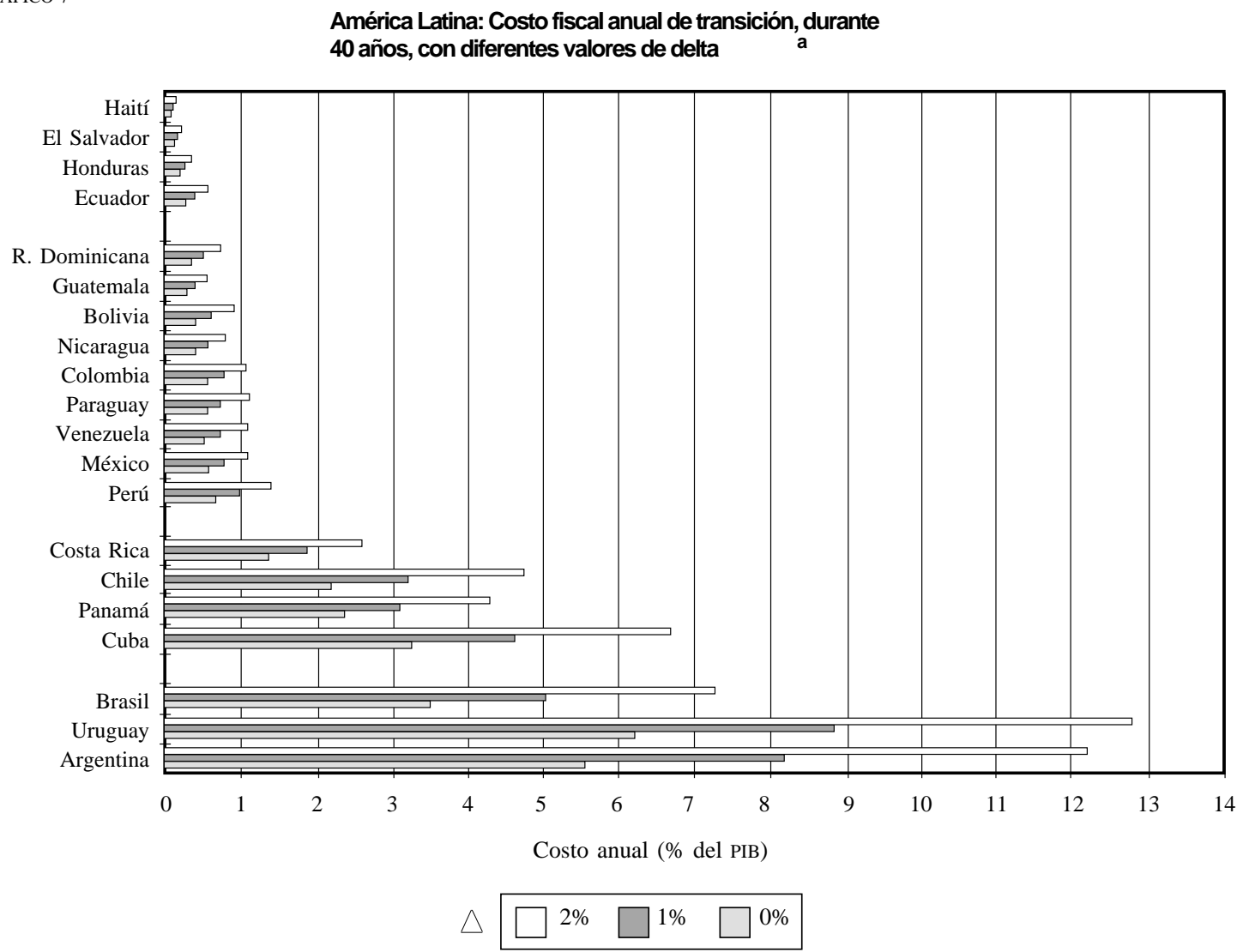

Fuente: Bravo y Uthoff (1999, cuadro 2).

a Delta es la diferencia entre la tasa de descuento y la tasa de crecimiento salarial.

una suma alzada, como en Chile y Colombia, se pueden obtener ciertas reducciones del costo fiscal anual en situaciones donde la tasa de decuento (i) supere a la tasa de crecimiento de la economía $(g)$. Sin embargo, estas reducciones no son tan sustanciales como cabría suponer: si $i$ supera a $g$ en dos puntos porcentuales, y se extiende el período de pago de 40 a 60 años (suponiendo que esto sea viable), el costo anual de reembolsar la deuda de $D=100 \%$ del PIB se reduciría de $3.7 \%$ a $2.9 \%$ del PIB, y si se prolongara a 80 años, el costo anual seguiría siendo todavía de $2.5 \% .^{28}$

Bravo y Uthoff (1999) examinan las trayectorias observadas y las proyectadas de los déficit transicionales de Chile, Argentina y Bolivia según el mo-

\footnotetext{
28 Después de unos noventa años, no se obtendrían más reducciones del costo fiscal anual; en general las reducciones son menores y el tiempo a partir del cual no se obtienen más reducciones es más corto mientras mayor es la diferencia $i-g$.
}

delo mencionado, señalando las restricciones que ellas implican para el presupuesto general del gobierno y el riesgo de incumplimiento con los derechos adquiridos por los trabajadores y jubilados. Un relato más detallado de los casos de Brasil y Chile se presenta en Uthoff (1999) y Arenas (2000), respectivamente, donde se muestra cómo el perfil temporal de la amortización de las obligaciones depende de la distribución por edad y sexo de la población activa y jubilada, presente y proyectada. En las simulaciones para Brasil, la característica más destacada es la mayor importancia relativa de las pensiones en curso de pago del sistema preexistente durante las etapas iniciales de la transición, y el predominio ulterior de las garantías estatales del sistema de reparto y los "bonos de reconocimiento", estos últimos otorgados a cohortes relativamente numerosas de personas en edad de trabajar. En el caso de Chile, tal vez el resultado más significativo sea la creciente importancia relativa de las mujeres y las 
garantías de pensiones mínimas y asistenciales en el mediano a largo plazo, que tenderá a mantener una gran responsabilidad fiscal en el financiamiento de las pensiones, mucho más allá del período de transición.

Cabe formular, por último, dos observaciones de orden práctico en el proceso de toma de decisiones. Primero, ni el tamaño de la deuda ni el programa de amortización están absolutamente determinados, y los países pueden y deben buscar diversas variantes para hacer más sustentables los costos fiscales (CEPAL, 1998; Queisser, 1998). En este sentido, muchos países latinoamericanos han realizado un viraje sólo parcial al sistema de capitalización (en todas las reformas recientes, salvo en Chile, México y Bolivia), lo que tiene especial importancia en países con sistemas de pensiones maduros, de alta cobertura y con poblaciones envejecidas, como Argentina y Uruguay. Se han introducido también cambios en los beneficios y las condi- ciones de elegibilidad simultáneamente con el viraje hacia la capitalización, así como otras cláusulas que buscan reducir las obligaciones fiscales (Holzmann, 1997; CEPAL, 1998; Mesa-Lago, 2000). En segundo lugar, el contexto macroeconómico y fiscal general es clave para la posibilidad de absorber los costos de transición. Por ejemplo, la puesta en marcha de la reforma chilena (de sustitución completa) ha sido posible por un desempeño macroeconómico muy favorable durante casi toda la transición, por la introducción activa de las adaptaciones necesarias al marco regulador del mercado financiero (Arrau, 1994), y ha requerido un importante esfuerzo de disciplina fiscal. Todos los países de la región que han hecho reformas han desplegado esfuerzos en ese sentido, con mayor o menor facilidad y equilibrio entre la reducción del costo fiscal y la reducción del bienestar de los participantes (Bravo y Uthoff, 1999 y Mesa-Lago, 2000).

\section{VI}

\section{Resumen y conclusiones}

La población de América Latina está comenzando a envejecer, luego de décadas de índices demográficos declinantes. Aunque situada todavía a buena distancia de los países más envejecidos del mundo, se proyecta que la región envejecerá con mucha mayor rapidez de lo que lo hicieron las sociedades actualmente más desarrolladas. Y estas transformaciones ocurrirán en contextos económicos e institucionales en que el nivel de vida de las generaciones de mayor edad estará mucho menos protegido que hoy en los países más desarrollados. Por lo tanto, es de suma importancia que los países de la región comiencen a evaluar las distintas opciones de política, para llegar a decisiones y programas que sean más apropiados para sus condiciones demográficas y económicas actuales y en el futuro cercano.

A menudo se postula que el envejecimiento de la población es una causa subyacente clave de los problemas financieros del sistema, pero el examen de los hechos enseña que generalmente esto no es así. Este artículo demuestra que la estructura por edades y los patrones de mortalidad de la población pueden tener efectos importantes sobre la evolución de mediano y largo plazo de los sistemas, pero que, comparativamente, las condiciones del mercado laboral y los problemas administrativos han desempeñado un papel mu- cho más decisivo, al menos hasta ahora. Los efectos más fuertes del envejecimiento de la población sobre los sistemas sobrevendrán en las próximas temporadas en una gran mayoría de países de la región, como se señala en la sección III de este artículo. En esa sección se examinó el comportamiento de varios indicadores del sistema de pensiones sobre el proceso de envejecimiento, que abarca desde las estructuras etarias más jóvenes en los países latinoamericanos de hoy hasta la distribución etaria proyectada de Francia para mediados del siglo XXI.

Este artículo ilustra cuáles podrían ser las tendencias generales del gasto en pensiones, el equilibrio financiero, el nivel de la deuda pensional y la rentabilidad del sistema de pensiones, de mantenerse las condiciones del sistema, del mercado laboral y de la economía. El análisis sugiere que, en escenarios futuros de envejecimiento y de normas invariables en el sistema, los países latinoamericanos podrían llegar a niveles de gasto y tal vez también de déficit cercanos a los de los países más desarrollados actualmente. Sin embargo, se vio que es muy difícil definir los niveles de gasto "sustentables" o "no sustentables" de las obligaciones pensionales tanto en términos generales como en un país determinado, excepto en unos pocos casos 
más bien extremos. El creciente nivel de gasto, debido en parte al envejecimiento de la población en el largo plazo, no debe considerarse como el síntoma de una crisis inminente o de una bancarrota, en la medida en que refleja una reasignación natural de recursos en el ciclo de vida, vinculada a una mayor longevidad de los individuos y de las cohortes. Lo mismo ocurre en el caso de las obligaciones pensionales, que tienden a ser naturalmente más elevadas en países con poblaciones más envejecidas, y con sistemas de pensiones más maduros y de cobertura más amplia. Cuando se requieren adaptaciones, los cambios paramétricos para compensar los efectos del envejecimiento de la población pueden ser adecuados en muchos contextos. En caso contrario, las opciones de reforma deben evaluarse considerando las múltiples alternativas posibles de esquemas públicos, privados o mixtos, de sistemas definidos por beneficios o por contribuciones, y de esquemas de capitalización o de reparto.

La introducción de una mayor capitalización de las contribuciones y los beneficios es un tipo de reforma que ha sido implementada en varios países latinoamericanos, y que se discute activamente también en otras regiones. En las secciones IV y V del presente artículo se plantean y analizan algunos de los temas respecto a este tipo de reforma. Señalamos que, aunque la capitalización generalmente se justifica por su potencial para reducir la presión sobre los presupuestos públicos, aumentar la eficiencia del mercado laboral y el desarrollo del mercado financiero, incrementar el ahorro, la acumulación de capital y el crecimiento, y ofrecer protección contra el envejecimiento de la población y las fluctuaciones de la estructura etaria, la teoría no resulta decisiva respecto de estas cuestiones.

Los datos empíricos tampoco son concluyentes, excepto en unos pocos aspectos específicos. El artículo examina un efecto directo del cambio al sistema de capitalización, a saber, el de la deuda pensional implícita que debe hacerse explícita, así como los costos fiscales que ello implica. El modelo utilizado, que considera la estructura por edades de la población, muestra el papel importante que ella desempeña. Se muestra también el menor efecto de la mortalidad en edad avanzada así como aquel más sustancial de la cobertura poblacional del sistema, los factores del mercado laboral y otros parámetros del sistema como las tasas de contribución y reemplazo. Los países con envejecimiento intermedio a más avanzado y sistemas con una amplia cobertura poblacional muestran niveles elevados de deuda pensional, que es a menudo mayor que el valor de su deuda pública oficial e incluso que su PIB anual. Para los países cuyas reformas apuntan a la capitalización plena, esto se traduce en costos fiscales de transición considerables durante los aproximadamente 40 años que se requieren para poner término a la transición de un tipo de sistema a otro.

Se señaló también que ni el tamaño de la deuda explicitada ni el programa de amortización están absolutamente determinados, puesto que pueden ser, y de hecho han sido, configurados en función de cada situación nacional. Los costos fiscales dependen de las estrategias que siguen los gobiernos respecto al grado de capitalización que se introduce y la forma en que se explicita la deuda pensional implícita. Los países han seguido diversas variantes, que tienden a hacer más tolerable el pago de los costos de transición, aunque afectan a veces negativamente el bienestar de los participantes en el sistema.

Entre las diversas opciones de reforma que se debaten actualmente, una que merece mencionarse es la que introduce mecanismos definidos por contribuciones en los sistemas de reparto. Este esquema permite la capitalización ("nocional") de las contribuciones a una tasa equivalente a la de crecimiento de la masa salarial, brindando beneficios actuarialmente justos entre las cohortes, sin necesidad de introducir la capitalización. Tal como en el sistema de capitalización, en este esquema los beneficios se ajustan, en gran medida endógenamente, a los cambios de la supervivencia en la edad avanzada y al envejecimiento de la población. Puesto que los regímenes de capitalización están definidos casi siempre por contribuciones, pueden integrarse con mayor facilidad en la clase de esquema descrito. Una opción de este tipo se está introduciendo actualmente en Brasil, y podría ser una alternativa interesante para otros países latinoamericanos que confrontan algunas de las restricciones demográficas, políticas o fiscales aquí analizadas.

(Traducido del inglés) 
APENDICE 1

\section{Datos sobre el gasto y el balance financiero en los sistemas de pensiones, 1990-1993}

Las estimaciones que se presentan en el cuadro A1 están basadas en los datos que aparecen en The cost of social security. Basic tables 1990-1993 (OIT, 1997). Las cifras correspondientes a gasto e ingresos pensionales se refieren a las pensiones de vejez, supervivencia e invalidez del sector público y privado. Se excluyen los renglones de gasto correspondientes a asignaciones familiares, indemnizaciones por desempleo, accidentes del trabajo, y gastos mortuorios, de enfermedad y maternidad, así como la columna "transferencias a otros esquemas". En general, se utilizaron los mismos renglones para el cálculo de los ingresos, así como también la exclusión de la columna "transferencias desde otros esquemas".

A continuación, se detallan las partidas incluidas y excluidas en cada país considerado ${ }^{\mathrm{a}} \mathrm{Se}$ han mantenido las referencias a las secciones e ítemes de los cuadros de la OIT citados, para facilitar posibles consultas.

\begin{tabular}{|c|c|c|}
\hline País & Gasto & Ingresos \\
\hline Argentina & 1. Pensiones 2. Pensiones NC & $\begin{array}{l}\text { 1. Pensiones 2. Pensiones NC 3. Poder judicial } \\
\text { 4. Retención salarial 5. Trabaj. por cuenta propia }\end{array}$ \\
\hline Bahamas & 1. Vejez, supervivencia y jubilación & 1. Vejez, supervivencia y jubilación \\
\hline Barbados & 1. VSI & 1. Pensiones $(1$ a $3-$ gasto por 2 y 3$)$ \\
\hline Belice & 1. VSI (largo plazo - asignación mortuoria) & 1. VSI (largo plazo - asign. mortuoria) \\
\hline Bolivia & 1. VSI + 2. c, d, f & 1. VSI + 2. c, d, f \\
\hline Chile & 1. VS ó TAOE & 1. VS - TDOE \\
\hline Colombia & Todo $-\mathrm{AT}-\mathrm{EM}-\mathrm{TAOE}$ & Todo $-\mathrm{AT}-\mathrm{EM}-\mathrm{TAOE}$ \\
\hline Costa Rica & 2. VSI & 2. VSI \\
\hline Cuba & 1. VSI & 1. VSI \\
\hline Dominica & 1. VSI (largo plazo — asignación mortuoria) & 1. VSI (largo plazo - asign. mortuoria) \\
\hline Ecuador & 1. VSI-EM-AT-DES & 1. VSI-EM-AT-DES \\
\hline Granada & 1. VSI + 5. Empleados públicos & 1. VSI + 5. Empleados públicos \\
\hline Guatemala & 1. VSI & 1. VSI \\
\hline Guyana & $\begin{array}{l}\text { 1.c VSI + 1.a.ii invalidez + 1.a.iii muerte } \\
+2 \text {. Fondo de dependientes }\end{array}$ & $\begin{array}{l}\text { 1.c VSI + 1.a.ii invalidez + 1.a.iii muerte }+2 \text {. Fondo } \\
\text { de dependientes }\end{array}$ \\
\hline Jamaica & $\begin{array}{l}\text { I. Seguro nacional — maternidad — asignación } \\
\text { mortuoria + III. Empleados públicos }\end{array}$ & $\begin{array}{l}\text { I. Seguro nacional — maternidad y asignación } \\
\text { mortuoria + III. Empleados públicos }\end{array}$ \\
\hline Nicaragua & 1. VSI & 1. VSI \\
\hline Panamá & 1. VSI + 2. Empleados públicos +4 . Administración & 1.VSI + 2. Empleados públicos + 4. Administración \\
\hline Trinidad y Tabago & 1. VSI +2 . Pensión de vejez NC & $\begin{array}{l}\text { 1. Seguro nacional — asignación mortuoria y gastos } \\
\text { de EM }\end{array}$ \\
\hline
\end{tabular}

${ }^{\text {a }}$ Las abreviaturas utilizadas son las siguientes:

\begin{tabular}{|c|c|c|c|c|}
\hline$\Delta \mathrm{T}$ & accidentes del trabajo & & $=$ gasto & TDOE $=$ Transferencias desde otros esquemas \\
\hline & desempleo & $\mathrm{NC}$ & $=$ no contributivas & $=$ vejez $\mathrm{y}$ supervivencia \\
\hline & $=$ enfermedad $\mathrm{y}$ maternidad & TAOE & $=$ Transferencias a otros esquemas. & $=$ vejez, supervivencia e invalidez \\
\hline
\end{tabular}

$\mathrm{EM}=$ enfermedad $\mathrm{y}$ maternidad

$\mathrm{NC}=$ no contributivas

$\mathrm{TAOE}=$ Transferencias a otros esquemas.
$\mathrm{VS} \quad=$ vejez $\mathrm{y}$ supervivencia

VSI $=$ vejez, supervivencia e invalidez 
CUADRO A.1

América Latina y el Caribe: Datos y estimaciones de gasto

y balance financiero de la seguridad social, 1990-1993

\begin{tabular}{|c|c|c|c|c|c|c|c|c|c|c|c|c|}
\hline \multirow[b]{3}{*}{ País } & \multirow[b]{3}{*}{$\begin{array}{c}\text { Relación de } \\
\text { dependencia (1990) }\end{array}$} & \multicolumn{5}{|c|}{$\begin{array}{l}\text { A. Estimación básica, incluida } \\
\text { la contribución estatal } \\
\text { (Porcentaje del PIB) }\end{array}$} & \multicolumn{6}{|c|}{$\begin{array}{l}\text { B. Estimación excluida la } \\
\text { contribución estatal } \\
\text { (Porcentaje del PIB) }\end{array}$} \\
\hline & & \multirow[b]{2}{*}{ Año } & \multicolumn{2}{|c|}{ Gasto } & \multicolumn{2}{|c|}{ Ingreso } & \multicolumn{2}{|c|}{ Balance } & \multicolumn{2}{|c|}{ Ingreso } & \multicolumn{2}{|c|}{ Balance } \\
\hline & & & Pensiones & Total & Pensiones & Total & Pensiones & Total & Pensiones & Total & Pensiones & Total \\
\hline Barbados & 0.30 & 1992 & 2.48 & 5.23 & 3.86 & 8.21 & 1.38 & 2.98 & 3.86 & 8.21 & 1.38 & 2.98 \\
\hline Argentina & 0.27 & 1992 & 4.41 & 4.78 & 4.71 & 5.28 & 0.30 & 0.50 & 4.52 & 5.09 & 0.11 & 0.31 \\
\hline Dominica & 0.23 & 1993 & 1.42 & 2.66 & 2.78 & 4.51 & 1.36 & 1.85 & 2.70 & 4.51 & 1.28 & 1.85 \\
\hline Jamaica & 0.21 & 1991 & 0.66 & 1.05 & 1.39 & 1.72 & 0.73 & 0.67 & 1.39 & 1.72 & 0.73 & 0.67 \\
\hline Cuba & 0.21 & 1991 & 8.40 & 18.00 & 8.40 & 18.00 & 0.00 & 0.00 & 4.85 & 6.50 & -3.55 & -11.50 \\
\hline Granada & 0.21 & 1993 & 2.23 & 2.66 & 4.14 & 4.83 & 1.91 & 2.17 & 4.14 & 4.83 & 1.91 & 2.17 \\
\hline Chile $^{\mathrm{a}}$ & 0.17 & 1993 & 6.89 & 22.67 & 18.16 & 34.15 & 11.27 & 11.48 & 13.16 & 16.25 & 6.27 & -6.42 \\
\hline Trinidad y Tabago & 0.17 & 1991 & 0.64 & 0.95 & 1.50 & 1.54 & 0.86 & 0.59 & 1.50 & 1.54 & 0.86 & 0.59 \\
\hline Panamá & 0.16 & 1990 & 5.76 & 9.31 & 6.01 & 10.43 & 0.25 & 1.12 & 5.66 & 10.08 & -0.10 & 0.77 \\
\hline Belice & 0.16 & 1992 & 0.24 & 1.14 & 1.38 & 2.65 & 1.14 & 1.51 & 1.38 & 2.65 & 1.14 & 1.51 \\
\hline Ecuador & 0.14 & 1991 & 1.85 & 1.95 & 2.39 & 2.93 & 0.54 & 0.98 & 2.13 & 2.67 & 0.28 & 0.72 \\
\hline Bolivia & 0.14 & 1992 & 1.72 & 1.83 & 2.01 & 2.23 & 0.29 & 0.40 & 1.94 & 2.16 & 0.22 & 0.33 \\
\hline Colombia & 0.13 & 1992 & 3.26 & 4.36 & 3.92 & 5.18 & 0.66 & 0.82 & 3.88 & 5.14 & 0.62 & 0.78 \\
\hline Costa Rica & 0.13 & 1993 & 1.92 & 7.65 & 2.26 & 8.91 & 0.34 & 1.26 & 2.19 & 8.08 & 0.27 & 0.43 \\
\hline Bahamas & 0.13 & 1992 & 1.72 & 2.32 & 2.80 & 3.43 & 1.08 & 1.11 & 2.67 & 3.30 & 0.95 & 0.98 \\
\hline Guatemala & 0.13 & 1993 & 0.28 & 1.05 & 0.49 & 1.35 & 0.21 & 0.30 & 0.49 & 1.35 & 0.21 & 0.30 \\
\hline Guyana & 0.13 & 1992 & 1.05 & 1.52 & 1.77 & 2.26 & 0.72 & 0.74 & 1.77 & 2.26 & 0.72 & 0.74 \\
\hline Nicaragua & 0.11 & 1992 & 0.22 & 0.50 & 0.22 & 0.53 & 0.00 & 0.03 & 0.21 & 0.47 & -0.01 & -0.03 \\
\hline Promedio & 0.17 & & 2.51 & 4.98 & 3.79 & 6.56 & 1.28 & 1.58 & 3.25 & 4.82 & 0.74 & -0.16 \\
\hline
\end{tabular}

Fuente: Estimaciones del autor, basadas en OIT (1997). Los indicadores demográficos se basan en estimaciones de población de CEPAL/ CELADE (1999).

a El "ingreso del capital" se excluyó en las estimaciones A y B, para fines de comparabilidad con los países que no habían iniciado sus "pilares" de capitalización en el período 1990-1993. 
APENDICE 2

\section{Ecuaciones del modelo}

El siguiente conjunto de ecuaciones se utiliza en este artículo para estudiar la relación entre un índice estándar de envejecimiento en el análisis del sistema de pensiones, la relación de dependencia de la edad avanzada, es decir, la relación entre la población en edad de jubilación y la población en edad de trabajar, e indicadores seleccionados del sistema de pensiones. Las ecuaciones están basadas en el supuesto de que la tasa de cotización $(c)$, la tasa de reemplazo $(r)$, así como la relación entre trabajadores cubiertos por el sistema de pensiones y la fuerza del trabajo asalariada $(k)$ se mantienen constantes en el tiempo en el país en cuestión. Si se supone además que las pensiones se calculan como una proporción de los salarios reales, se desprende que:

1. El gasto del sistema de pensiones $(E)$, expresado como una fracción del PIB $(Y)$ es:

$$
E=r \cdot s \cdot k \cdot d
$$

donde $s$ es la proporción que representa la masa salarial en el PIB.

2. Puesto que el ingreso del sistema por concepto de contribuciones es $C=c \cdot s \cdot k$, el balance financiero del sistema $(C-E)$, expresado como cociente del PIB es:

$$
F i=s \cdot k \cdot(c-r d)
$$

y el balance expresado como una fracción del ingreso es:

$$
F_{i i}=1-\left(\frac{r}{c}\right) d
$$

3. La deuda pensional implícita (véase Bravo y Uthoff, 1999) está dada por:

$$
D=s k\left(c A_{a}+r d A_{r}\right)
$$

donde $A_{a}$ es el promedio actualizado de años de cotizaciones de los económicamente activos en un momento dado, y $A$ es el promedio de años actualizado de la esperanza de vida de todos aquellos en edad de jubilación en un momento dado.

4. La tasa de retorno ( $\rho$ ) del sistema de pensiones, cuando las reglas del sistema son fijas durante toda la vida adulta de la cohorte (Bravo, 1996, p. 126), puede escribirse así:

$$
\rho_{i}=\frac{1}{\left(A_{R}-A_{W}\right)}\left[\ln \left(\frac{r}{c}\right)+\ln \left(\frac{L R}{L W}\right)\right]+\sigma
$$

siendo $A_{R}$ la edad media de jubilación, $A_{w}$ la edad media laboral, $L R$ el promedio de años vividos como jubilado, $L W$ el número de años laborales del individuo o cohorte en cuestión, y $\sigma$ la tasa de crecimiento salarial. Cuando impera la norma del equilibrio financiero (Bravo, 1996, p. 127), entonces

$$
\rho_{i i}=\frac{\ln \left(\frac{L R}{L W}\right)-\ln (d)}{\left(A_{R}-A_{W}\right)}+\sigma
$$

\section{Bibliografía}

Arenas, A. (2000): El sistema de pensiones en Chile: resultados y desafíos pendientes, Santiago de Chile, Comisión Económica para América Latina y el Caribe (CEPAL).

Arrau, P. (1991): La reforma previsional chilena y su financiamiento durante la transición, Colección estudios CIEPLAN, $\mathrm{N}^{\circ} 32$ Santiago de Chile, Corporación de Investigaciones Económicas para Latinoamérica (CIEPLAN)

(1994): Fondo de pensiones y desarrollo del mercado de capitales en Chile: 1980-1993, serie Financiamiento del desarrollo, $\mathrm{N}^{\circ} 19$, Santiago de Chile, CEPAL.

Auerbach, A. y J. Kotlikoff (1987): Dynamic Fiscal Policy, Cambridge, Massachusetts, Cambridge University Press.

Banco Mundial (1994): Averting the Old Age Crisis. Policy Research Report, Oxford, Reino Unido, Oxford University Press.

Baldacci, E. y D. Tuzi (1999): Effects of labour market changes on the sustainability of the Italian pension system, en Âge, génération et activité: Vers un nouveau contrat social? Actes, 1ères Rencontres Sauvy, París, Instituto Nacional de Estudios Demográficos (INED), diciembre.

Blanchet, D. (1990): Retraites par capitalisation et par répartition selon le contexte démographique: quelques résultats comparatifs, Annales d'économie et de statistique, $\mathrm{N}^{\circ} 18$.

(1998): Le débat repartition-capitalisation: un état des lieux, Retraites et épargne, París, Conseil d'analyse économique.

Bravo, J. (1992): Visiones teóricas de la transición de la fecundidad en América Latina. ¿Qué relevancia tiene un enfoque difusionista?, Notas de población, año XX, № 56 , Santiago de Chile, Centro Latinoamericano de Demografía (CELADE).

(1996): La tasa de retorno de los sistemas de pensiones de reparto, Estudios de economía, vol. 23, $\mathrm{N}^{\circ}$ 1, Santiago de
Chile, Universidad de Chile, Facultad de Ciencias Económicas y Administrativas, Departamento de Economía, junio.

(1999): Envejecimiento de la población y responsabilidades fiscales hacia los sistemas de pensiones en América Latina, Seminario Responsabilidades Fiscales en Sistemas de Previsión, Santiago de Chile, CEPAL, 2 y 3 de septiembre.

Bravo, J. y A. Uthoff (1999): Transitional Fiscal Costs and Demographic Factors, in Shifting from Unfunded to Funded Pensions in Latin America, serie Financiamiento del desarrollo, $\mathrm{N}^{\circ} 88$, Santiago de Chile, CEPAL.

Brasil, Gobierno (1998): Custos de transição para um sistema de capitalização individual, Informe de Previdência Social, vol. 10, $\mathrm{N}^{\circ} 2$, Brasilia, febrero.

Calot, G. y J. C. Chesnais (1997): Le vieillissement démographique dans l'Union européene à l'horizon 2050: une étude d'impact, Travaux et recherches de prospective, $\mathrm{N}^{\circ} 6$, París, Comisaría General del Plan, octubre.

CEPAL (1998): El pacto fiscal. Fortalezas, debilidades, desafíos, LC/G.2024, Santiago de Chile, abril.

CEPAL/CELADE (1996): Impacto de las tendencias demográficas sobre los sectores sociales en América Latina: contribución al diseño de políticas y programas, serie E-CELADE, $\mathrm{N}^{\circ} 45$, Santiago de Chile.

(1998): América Latina: proyeccciones de población, 19702050, Boletín demográfico, año 31, N 62, Santiago de Chile. (1999): Latin America: Economically active population 1980-2025, Demographic Bulletin, Nㅜ 64, Santiago de Chile.

Chackiel, J. (2000): El envejecimiento de la población latinoamericana, Encuentro latinoamericano y caribeño sobre las personas de edad, Santiago de Chile, CEPAL, agosto. 
Chand, S. y A. Jaeger (1996): Ageing Populations and Public Pension Schemes, Occasional paper, $\mathrm{N}^{\circ} 147$, Washington, D.C., Fondo Monetario Internacional (FMI).

Chauveau, T. y R. Loufir (1995): L'avenir des régimes publics de retraite dans les pays du G7, Revue de l'OFCE, $\mathrm{N}^{\circ} 52$, París, Observatoire français des conjonctures économiques (OFCE), enero.

Cifuentes, R. (1995): Reforma de los sistemas previsionales: aspectos macroeconómicos, Cuadernos de economía, año 32, № 96, Santiago de Chile, Pontificia Universidad Católica de Chile.

Conseil d'analyse économique (1998): Retraites et épargne, París, La documentation française.

Conte-Grand, A. (1995): Reparto o capitalización-gestión pública o privada: aporte para las discusiones en materia de seguridad social, Documento de trabajo, $\mathrm{N}^{\circ} 24$, Santiago de Chile, Organización Internacional del Trabajo (OIT).

Cosio-Zavala, M. (1996): The demographic transition in Latin America and Europe, J. M. Guzmán y otros eds., The Fertility Transition in Latin America, Oxford, Reino Unido, Clarendon Press.

Cox, A. (1999): Pensions projections for Chilean men and women: Estimates from social security contributions, World Bank Research Project on Gender and Social Security, Washington, D.C., Banco Mundial.

Dinh, Q. (1995): Projection de population totale pour la France Métropolitaine, base RP90, horizons 1990-2050, París, Instituto Nacional de Estadística y Estudios Económicos (INSEE).

Dumont, J. P. (1998): Les systèmes de protection sociale en Europe, París, Fédération mutualiste parisienne (FMP), Mutualité francilienne.

El Mercurio (1997): Pulso latinoamericano, Santiago de Chile, 27 de marzo.

Franco, D. (1995): Pension Liabilities: Their Use and Misuse in the Assessment of Fiscal Policies, Economic papers, N 110 , Bruselas, Luxemburgo, Comisión Europea, mayo.

Gokhale, J. (1995): Demographic changes, generational accounts and national saving in the United States. Trabajo presentado a la Conference on Intergenerational Economic Relations and Demographic Change, Honolulu, Hawaii, Unión Internacional para el Estudio Científico de la Población (UIECP)/Centro Este-Oeste, septiembre.

Guzmán, J. M. (1996): Social change and fertility decline in Latin America, J.M. Guzmán y otros, eds., The Fertility Transition in Latin America, Oxford, Reino Unido, Clarendon Press.

Holzmann, R. (1997): On Economic Benefits and Fiscal Requirements of Moving from Unfunded to Funded Pensions, serie Financiamiento del desarrollo, $\mathrm{N}^{\circ} 48$, Santiago de Chile, CEPAL.

(1999): Comments to "Rethinking pension reform: Ten myths about social security", by P. Orszag and J. Stiglitz. Trabajo presentado a la Conference on New Ideas about Old Age Security, Washington, D.C., Banco Mundial, septiembre.

Kinsella, K. y Y. Gist (1995): Older Workers, Retirement and Pensions: A comparative International Chartbook, Washington, D.C., U.S. Department of Commerce, Bureau of the Census/U.S. Department of Health and Human Services National Institute on Ageing.

Le Monde-Economie (1999): París, 19 de enero.

Lee, R. (1995): A Trans-cultural perspective of intergenerational transfers. Trabajo presentado a la Conference on Intergenerational Economic Relations and Demographic Change, Honolulu, Hawaii, UIEP/Centro Este-Oeste, septiembre.

Leibfritz, W. y D. Roseveare (1995): Ageing populations and government budgets, The OECD Observer, $\mathrm{N}^{\circ} 197$, París, Organización de Cooperación y Desarrollo Económicos (OCDE).

Mesa-Lago, C. (1985): Social Security in Latin America, Cuadernos de la CEPAL, $\mathrm{N}^{\circ} 85$, Santiago de Chile, CEPAL.
(1991): Social Security and Prospects for Equity in Latin America, World Bank discussion papers, $\mathrm{N}^{\circ} 140$, Washington, D.C., Banco Mundial.

(1994): La reforma de la seguridad social y las pensiones en América Latina: importancia y evaluación de las alternativas de privatización, serie Reformas de política pública, $\mathrm{N}^{\circ} 28$, Santiago de Chile, CEPAL.

(2000): Estudio comparativo de los costos fiscales en la transición de ocho reformas de pensiones en América Lati$n a$, serie Financiamiento del desarrollo, $\mathrm{N}^{\circ} 93$, Santiago de Chile, CEPAL.

Meslé, F. y J. Vallin (1998): Tables de mortalité du moment 1806 2100: sexe féminin, sexe masculin, sexes réunis, París, INED.

Naciones Unidas (1998): World Population Prospects: The 1996 Revision, Nueva York, Departamento de Asuntos Económi$\cos$ y Sociales.

(1999) World Population Prospects: The 1998 Revision. Sex and Age, Nueva York, Departamento de Asuntos Económicos y Sociales.

OCDE (1993): Ageing in OECD Countries: A Critical Policy Challenge, Social policy studies, $\mathrm{N}^{\circ} 20$, París.

OIT (1997): The cost of social security, Basic Tables 1990-1993, http:/www-ilo-mirror.who.or.jp/public/english/110secso/css/.

Orszag, P. y J. Stiglitz (1999): Rethinking pension reform: Ten myths about social security. Trabajo presentado a la Conference on New Ideas about Old Age Security, Washington, D.C., Banco Mundial, 14 y 15 de septiembre.

Pinheiro, V. y S. Vieira (2000): Reforma previsional en Brasil. La nueva regla para el cálculo de los beneficios, serie Financiamiento del desarrollo, $\mathrm{N}^{\circ} 97$, Santiago de Chile, CEPAL.

Queisser, M. (1998): The second-generation pension reforms in Latin America, OECD working paper, París, OCDE.

Reynaud, E. (coord.) (1998): Les retraites dans l'Union européene: adaptation aux évolutions économiques et sociales, París, L'Hartman.

Rofman, R. (1997): Modelling transition costs in social security reforms. Trabajo presentado a la International Population Conference, Beijing 1997, session I.29: Intergenerational Transfers and Population Ageing, Bélgica, UIECP.

Roseveare, D. y otros (1996): Ageing Populations, Pension Systems and Government Budgets: Simulations for 20 OECD Countries, OECD Economics Department working papers, $\mathrm{N}^{\circ} 168$, París, OCDE.

Sauvy, A. (1953): L'Europe et sa population, París, Institut National d'Études Démographiques.

Schmidt-Hebbel, K. (1995): La reforma pensional colombiana: efectos fiscales y macroeconómicos, Santafé de Bogotá, Bolsa de Bogotá.

(1997): Pension Reform, Informal Markets and Long-Term Income and Welfare, Documento de trabajo, $\mathrm{N}^{\circ} 4$, Santiago de Chile, Banco Central de Chile.

Thompson, L. (1999): Pension reform in industrialized countries. Trabajo presentado a Latin American and Caribbean Symposium on Older Persons, Santiago de Chile, CEPAL, 8 al 10 de septiembre.

Thogersen, O. (1998): A note on intergenerational risk sharing and the design of pay-as-you-go pension programs, Population Economics, vol. 111, № 3, Berlín, Springer-Verlag.

Uthoff, A. (1997): Reformas a los sistemas de pensiones, mercado de capitales y ahorro, Revista de la CEPAL, N 63, LC/G.1986$\mathrm{P}$, Santiago de Chile, CEPAL.

(1999): Perfil de responsabilidades fiscales de una eventual sustitución hacia un sistema de capitalización individual en Brasil. Trabajo presentado al Seminario Responsabilidades Fiscales en Sistemas de Previsión, Santiago de Chile, CEPAL, 2 y 3 de septiembre.

Uthoff, A. y J. Bravo (1998): Deuda previsional y privatización de los sistemas de pensiones, X Seminario Regional de Política 
Fiscal: Compilación de documentos, Santiago de Chile, CEPAL, 26 al 28 de enero.

Valdés-Prieto, S., ed. (1997): The Economics of Pensions: Principles, Polices and International Experience, Londres, Cambridge University Press.

Valdés-Prieto, S. (1999): El tamaño de las comisiones de las AFP: comparación internacional, Santiago de Chile, Pontificia Universidad Católica de Chile.

Van der Noord P. y P. Herd (1993): Pension Liabilities in Seven Major Economies, Working paper, $\mathrm{N}^{\circ} 142$, París, OCDE.

(1994): Estimating pension liabilities: A methodological framework, OECD Economic Studies, № 23, París, OCDE.
Vernière, L. (1997a): La mesure et l'interpretation de l'équivalent patrimoniale des droits à la retraite, Questions retraite, $\mathrm{N}^{\circ} 4$, París, Caisse de dépots et consignations, Branche retraites. (1997b): Une évaluation de l'équivalent patrimonial des droits à la retraite, Questions retraite, $\mathrm{N}^{\circ} 5$, París, Caisse de dépots et consignations, Branche retraites.

Villa, M. y L. Rivadeneira (2000): El proceso de envejecimiento de la población en América Latina y el Caribe: una expresión de la transición demográfica, Encuentro Latinoamericano y Caribeño sobre las Personas de Edad, Santiago de Chile, CEPAL, agosto. 